\title{
Adaptive BP-Dock: an Induced Fit Docking Approach for Full Receptor Flexibility
}

\author{
Ashini Bolia ${ }^{\#}$ and Sefika Banu Ozkan ${ }^{\dagger *}$ \\ "Department of Chemistry and Biochemistry, Arizona State University Tempe AZ, USA \\ 'Department of Physics, Center for Biological Physics, Arizona State University Tempe \\ $\mathrm{AZ}, \mathrm{USA}^{\dagger}$
}

\section{AUTHOR INFORMATION}

Corresponding Author

*Phone number: +1 480-965-2890; Email: banu.ozkan@asu.edu 
Table S1. The PDB codes of unbound and bound structures, the chain length, RMSDs between bound and unbound structures, the native ligands, for the 5 data sets and HIV-1 Reverse Transcriptase and Urokinase proteins used in the study.

\begin{tabular}{|c|c|c|c|c|c|}
\hline \multirow[b]{2}{*}{ Protein } & \multicolumn{2}{|c|}{ PDB Code } & \multirow[b]{2}{*}{ Chain Length } & \multirow{2}{*}{$\begin{array}{l}\text { RMSD } \\
\text { (̊̊) }\end{array}$} & \multirow[b]{2}{*}{ Ligand } \\
\hline & $\begin{array}{l}\text { Bound } \\
\text { (Holo) }\end{array}$ & $\begin{array}{c}\text { Unbound } \\
\text { (Apo) }\end{array}$ & & & \\
\hline HIV-Protease & $1 \mathrm{HBV}$ & $2 \mathrm{PC} 0 *$ & $198(\mathrm{~A}, \mathrm{~B})$ & 1.65 & SB203238 \\
\hline HIV-Protease & 1HEG & $2 \mathrm{PC} 0^{*}$ & $198(\mathrm{~A}, \mathrm{~B})$ & 1.614 & SKF 107457 \\
\hline HIV-Protease & $1 \mathrm{HIH}$ & $2 \mathrm{PC} 0 *$ & $198(\mathrm{~A}, \mathrm{~B})$ & 1.343 & CGP-53820 \\
\hline HIV-Protease & 1HIV & $2 \mathrm{PC} 0^{*}$ & $198(\mathrm{~A}, \mathrm{~B})$ & 1.422 & U75875 \\
\hline HIV-Protease & 1HPS & $2 \mathrm{PC} 0 *$ & $198(\mathrm{~A}, \mathrm{~B})$ & 1.606 & SB206343 \\
\hline HIV-Protease & 1HTE & $2 \mathrm{PC} 0 *$ & $198(\mathrm{~A}, \mathrm{~B})$ & 1.505 & GR123976 \\
\hline HIV-Protease & $1 \mathrm{HTF}$ & $2 \mathrm{PC} 0 *$ & $198(\mathrm{~A}, \mathrm{~B})$ & 1.436 & GR126045 \\
\hline HIV-Protease & 1HTG & $2 \mathrm{PC} 0^{*}$ & $198(\mathrm{~A}, \mathrm{~B})$ & 1.405 & GR137615 \\
\hline HIV-Protease & 1HVI & $2 \mathrm{PC} 0 *$ & $198(\mathrm{~A}, \mathrm{~B})$ & 1.344 & A77003 (R,S) \\
\hline HIV-Protease & $1 \mathrm{HVJ}$ & $2 \mathrm{PC} 0^{*}$ & $198(\mathrm{~A}, \mathrm{~B})$ & 1.347 & A78791 (S,-) \\
\hline HIV-Protease & $1 \mathrm{HVK}$ & $2 \mathrm{PC} 0 *$ & $198(\mathrm{~A}, \mathrm{~B})$ & 1.352 & A76928 (S,S) \\
\hline HIV-Protease & $1 \mathrm{HVL}$ & $2 \mathrm{PC} 0 *$ & $198(\mathrm{~A}, \mathrm{~B})$ & 1.347 & A76889 $(\mathrm{R}, \mathrm{R})$ \\
\hline HIV-Protease & 1HVS & $2 \mathrm{PC} 0 *$ & $198(\mathrm{~A}, \mathrm{~B})$ & 1.406 & A77 \\
\hline HIV-Protease & $1 \mathrm{SBG}$ & $2 \mathrm{PC} 0 *$ & $198(\mathrm{~A}, \mathrm{~B})$ & 1.561 & IM1 \\
\hline HIV-Protease & $4 \mathrm{HVP}$ & $2 \mathrm{PC} 0 *$ & $198(\mathrm{~A}, \mathrm{~B})$ & 1.538 & MVT-101 \\
\hline HIV-Protease & $4 \mathrm{PHV}$ & $2 \mathrm{PC} 0 *$ & $198(\mathrm{~A}, \mathrm{~B})$ & 1.373 & L-700, 417 \\
\hline HIV-Protease & $5 \mathrm{HVP}$ & $2 \mathrm{PC} 0 *$ & $198(\mathrm{~A}, \mathrm{~B})$ & 1.427 & $\begin{array}{c}\text { ACETYL- } \\
\text { PEPSTATIN } \\
\end{array}$ \\
\hline HIV-Protease & $9 \mathrm{HVP}$ & $2 \mathrm{PC} 0 *$ & $198(\mathrm{~A}, \mathrm{~B})$ & 1.512 & A-74704 \\
\hline HIV-Protease & $1 \mathrm{~A} 30$ & $2 \mathrm{PC} 0 *$ & $198(\mathrm{~A}, \mathrm{~B})$ & 1.49 & Tri-peptide \\
\hline HIV-Protease & $1 \mathrm{KZK}$ & $2 \mathrm{PC} 0^{*}$ & $198(\mathrm{~A}, \mathrm{~B})$ & 1.253 & JE-2147 \\
\hline Carbonic Anhydrase II & 1OQ5 & 2ILI & 259 & 0.134 & Celecoxib \\
\hline Carbonic Anhydrase II & $1 \mathrm{AVN}$ & 2ILI & 259 & 0.254 & Histamine \\
\hline Carbonic Anhydrase II & $1 \mathrm{CIL}$ & 2ILI & 259 & 0.239 & ETS \\
\hline Carbonic Anhydrase II & $1 \mathrm{CIM}$ & 2ILI & 259 & 0.258 & PTS \\
\hline Carbonic Anhydrase II & $1 \mathrm{CIN}$ & 2ILI & 259 & 0.244 & MTS \\
\hline Carbonic Anhydrase II & $1 \mathrm{CNW}$ & 2ILI & 259 & 0.231 & EG1 \\
\hline Carbonic Anhydrase II & $1 \mathrm{CNX}$ & 2ILI & 259 & 0.248 & EG2 \\
\hline Carbonic Anhydrase II & $1 \mathrm{CNY}$ & 2ILI & 259 & 0.258 & EG3 \\
\hline Carbonic Anhydrase II & $1 \mathrm{OKL}$ & 2ILI & 259 & 0.255 & MNS \\
\hline Alcohol Deydrogenase & $1 \mathrm{ADB}$ & $8 \mathrm{ADH}$ & 374 & 1.218 & CND \\
\hline Alcohol Deydrogenase & $1 \mathrm{ADC}$ & $8 \mathrm{ADH}$ & 374 & 1.219 & PAD \\
\hline
\end{tabular}




\begin{tabular}{|c|c|c|c|c|c|}
\hline Alcohol Deydrogenase & $1 \mathrm{ADF}$ & $8 \mathrm{ADH}$ & 374 & 0.33 & TAD \\
\hline Alcohol Deydrogenase & 1BTO & $8 \mathrm{ADH}$ & 374 & 1.137 & SSB \\
\hline Alcohol Deydrogenase & 1HLD & $8 \mathrm{ADH}$ & 374 & 1.135 & $\mathrm{PFB}, \mathrm{NAD}$ \\
\hline Alcohol Deydrogenase & $1 \mathrm{LDE}$ & $8 \mathrm{ADH}$ & 374 & 1.148 & FPI,NAD \\
\hline Alcohol Deydrogenase & $1 \mathrm{LDY}$ & $8 \mathrm{ADH}$ & 374 & 1.152 & CXF,NAD \\
\hline Alcohol Deydrogenase & 3ВТО & $8 \mathrm{ADH}$ & 374 & 1.16 & $\mathrm{SSB}, \mathrm{NAD}$ \\
\hline Alpha-Thrombin & $1 \mathrm{~A} 4 \mathrm{~W}$ & $1 \mathrm{C} 5 \mathrm{~L}$ & 274(L,H,I) & 0.351 & QWE \\
\hline Alpha-Thrombin & 1AE8 & $1 \mathrm{C} 5 \mathrm{~L}$ & 298(L,H,I) & 0.331 & AZL \\
\hline Alpha-Thrombin & $1 \mathrm{BMM}$ & $1 \mathrm{C} 5 \mathrm{~L}$ & 295(L,H,I) & 0.455 & BMS-186282 \\
\hline Alpha-Thrombin & $1 \mathrm{BMN}$ & $1 \mathrm{C} 5 \mathrm{~L}$ & 292(L,H,I) & 0.423 & BMS-189090 \\
\hline Alpha-Thrombin & 1D3D & $1 \mathrm{C} 5 \mathrm{~L}$ & $290(\mathrm{~L}, \mathrm{H}, \mathrm{I})$ & 0.352 & BZT \\
\hline Alpha-Thrombin & 1D3P & $1 \mathrm{C} 5 \mathrm{~L}$ & $290(\mathrm{~L}, \mathrm{H}, \mathrm{I})$ & 0.317 & BT3 \\
\hline Alpha-Thrombin & 1D4P & $1 \mathrm{C} 5 \mathrm{~L}$ & $290(\mathrm{~L}, \mathrm{H}, \mathrm{I})$ & 0.333 & $\mathrm{BPP}$ \\
\hline Alpha-Thrombin & 1DWB & $1 \mathrm{C} 5 \mathrm{~L}$ & $298(\mathrm{~L}, \mathrm{H}, \mathrm{I})$ & 0.386 & Benzyldiamine \\
\hline Alpha-Thrombin & 1DWC & $1 \mathrm{C} 5 \mathrm{~L}$ & $298(\mathrm{~L}, \mathrm{H}, \mathrm{I})$ & 0.386 & $\begin{array}{c}\text { MD-805 } \\
\text { (Argatroban) } \\
\end{array}$ \\
\hline Alpha-Thrombin & 1DWD & $1 \mathrm{C} 5 \mathrm{~L}$ & $298(\mathrm{~L}, \mathrm{H}, \mathrm{I})$ & 0.378 & NAPAP \\
\hline Alpha-Thrombin & 1HDT & $1 \mathrm{C} 5 \mathrm{~L}$ & $303(\mathrm{~L}, \mathrm{H}, \mathrm{I})$ & 0.511 & BMS-183507 \\
\hline Alpha-Thrombin & 1UVS & $1 \mathrm{C} 5 \mathrm{~L}$ & $268(\mathrm{~L}, \mathrm{H}, \mathrm{I})$ & 0.479 & BM51.1011 \\
\hline Alpha-Thrombin & $10 Y T$ & $1 \mathrm{C} 5 \mathrm{~L}$ & $305(\mathrm{~L}, \mathrm{H}, \mathrm{I})$ & 0.387 & FSN \\
\hline Cytohchrome C Peroxidase & $1 \mathrm{AC} 4$ & $1 \mathrm{CCP} *$ & 291 & 0.286 & TMT \\
\hline Cytohchrome C Peroxidase & $1 \mathrm{AC} 8$ & $1 \mathrm{CCP} *$ & 291 & 0.299 & TMZ \\
\hline Cytohchrome C Peroxidase & $1 \mathrm{AEB}$ & $1 \mathrm{CCP} *$ & 291 & 0.299 & $3 \mathrm{MT}$ \\
\hline Cytohchrome C Peroxidase & 1AED & $1 \mathrm{CCP} *$ & 291 & 0.299 & DTI \\
\hline Cytohchrome C Peroxidase & $1 \mathrm{AEE}$ & $1 \mathrm{CCP} *$ & 291 & 0.309 & ANL \\
\hline Cytohchrome C Peroxidase & $1 \mathrm{AEF}$ & $1 \mathrm{CCP} *$ & 291 & 0.299 & $3 \mathrm{AP}$ \\
\hline Cytohchrome C Peroxidase & $1 \mathrm{AEG}$ & $1 \mathrm{CCP} *$ & 291 & 0.299 & $4 \mathrm{AP}$ \\
\hline Cytohchrome C Peroxidase & $1 \mathrm{AEH}$ & $1 \mathrm{CCP} *$ & 291 & 0.299 & $24 \mathrm{~T}$ \\
\hline Cytohchrome C Peroxidase & $1 \mathrm{AEJ}$ & $1 \mathrm{CCP} *$ & 291 & 0.299 & NVI \\
\hline Cytohchrome C Peroxidase & $1 \mathrm{AEK}$ & $1 \mathrm{CCP} *$ & 291 & 0.299 & IDM \\
\hline Cytohchrome C Peroxidase & $1 \mathrm{AEM}$ & $1 \mathrm{CCP} *$ & 291 & 0.299 & MPI \\
\hline Cytohchrome C Peroxidase & $1 \mathrm{AEN}$ & $1 \mathrm{CCP} *$ & 291 & 0.286 & $25 \mathrm{~T}$ \\
\hline Cytohchrome C Peroxidase & $1 \mathrm{AEO}$ & $1 \mathrm{CCP} *$ & 291 & 0.286 & $2 \mathrm{AP}$ \\
\hline Cytohchrome C Peroxidase & $1 \mathrm{AEQ}$ & $1 \mathrm{CCP} *$ & 291 & 0.299 & $2 \mathrm{EZ}$ \\
\hline Cytohchrome C Peroxidase & $1 \mathrm{AES}$ & $1 \mathrm{CCP} *$ & 291 & 0.31 & IMD \\
\hline Cytohchrome C Peroxidase & $1 \mathrm{AET}$ & $1 \mathrm{CCP} *$ & 291 & 0.281 & $1 \mathrm{MZ}$ \\
\hline Cytohchrome C Peroxidase & $1 \mathrm{AEU}$ & $1 \mathrm{CCP} *$ & 291 & 0.286 & $2 \mathrm{MZ}$ \\
\hline Cytohchrome C Peroxidase & $1 \mathrm{AEV}$ & $1 \mathrm{CCP} *$ & 291 & 0.267 & AMT \\
\hline
\end{tabular}




\begin{tabular}{|c|c|c|c|c|c|}
\hline HIV-1 Reverse Transcriptase & 1DTQ & $1 \mathrm{DLO}$ & 556 & 2.948 & FPT \\
\hline HIV-1 Reverse Transcriptase & 1DTT & $1 \mathrm{DLO}$ & 556 & 2.612 & FTC \\
\hline HIV-1 Reverse Transcriptase & $1 \mathrm{IKV}$ & $1 \mathrm{DLO}$ & 556 & 2.574 & EFZ \\
\hline HIV-1 Reverse Transcriptase & $1 \mathrm{IKW}$ & $1 \mathrm{DLO}$ & 556 & 2.554 & EFZ \\
\hline HIV-1 Reverse Transcriptase & $1 \mathrm{IKX}$ & $1 \mathrm{DLO}$ & 556 & 2.419 & PNU \\
\hline HIV-1 Reverse Transcriptase & $1 \mathrm{IKY}$ & $1 \mathrm{DLO}$ & 556 & 2.413 & MSD \\
\hline HIV-1 Reverse Transcriptase & 1JLQ & $1 \mathrm{DLO}$ & 556 & 2.452 & SBN \\
\hline HIV-1 Reverse Transcriptase & $1 \mathrm{TKT}$ & $1 \mathrm{DLO}$ & 556 & 2.982 & $\mathrm{H} 12$ \\
\hline HIV-1 Reverse Transcriptase & $1 \mathrm{TKX}$ & $1 \mathrm{DLO}$ & 556 & 2.384 & GWB \\
\hline HIV-1 Reverse Transcriptase & $1 \mathrm{TKZ}$ & $1 \mathrm{DLO}$ & 556 & 3.002 & H16 \\
\hline HIV-1 Reverse Transcriptase & $1 \mathrm{TL} 1$ & $1 \mathrm{DLO}$ & 556 & 2.967 & $\mathrm{H} 18$ \\
\hline HIV-1 Reverse Transcriptase & 1TL3 & $1 \mathrm{DLO}$ & 556 & 2.947 & $\mathrm{H} 20$ \\
\hline HIV-1 Reverse Transcriptase & $1 \mathrm{TV} 6$ & $1 \mathrm{DLO}$ & 556 & 3.21 & СР9 \\
\hline HIV-1 Reverse Transcriptase & $2 \mathrm{~B} 5 \mathrm{~J}$ & $1 \mathrm{DLO}$ & 556 & 3.183 & $3 \mathrm{AC}$ \\
\hline HIV-1 Reverse Transcriptase & 2BAN & $1 \mathrm{DLO}$ & 556 & 3.516 & 357 \\
\hline HIV-1 Reverse Transcriptase & 2BE2 & $1 \mathrm{DLO}$ & 556 & 2.76 & $\mathrm{R} 22$ \\
\hline HIV-1 Reverse Transcriptase & $2 \mathrm{HNY}$ & $1 \mathrm{DLO}$ & 556 & 3.082 & NVP \\
\hline HIV-1 Reverse Transcriptase & $3 \mathrm{IOR}$ & $1 \mathrm{DLO}$ & 556 & 2.288 & RT3 \\
\hline HIV-1 Reverse Transcriptase & $3 \mathrm{IOS}$ & $1 \mathrm{DLO}$ & 556 & 2.074 & RT7 \\
\hline HIV-1 Reverse Transcriptase & 3IRX & $1 \mathrm{DLO}$ & 556 & 3.692 & UDR \\
\hline HIV-1 Reverse Transcriptase & 3 IS9 & $1 \mathrm{DLO}$ & 556 & 3.47 & $\mathrm{AC} 7$ \\
\hline HIV-1 Reverse Transcriptase & 3LP2 & $1 \mathrm{DLO}$ & 556 & 1.918 & LP9 \\
\hline HIV-1 Reverse Transcriptase & 3M8P & $1 \mathrm{DLO}$ & 556 & 2.173 & $65 \mathrm{~B}$ \\
\hline HIV-1 Reverse Transcriptase & $3 \mathrm{M} 8 \mathrm{Q}$ & $1 \mathrm{DLO}$ & 556 & 1.992 & DJZ \\
\hline HIV-1 Reverse Transcriptase & $3 \mathrm{QO} 9$ & $1 \mathrm{DLO}$ & 556 & 4.054 & QO9 \\
\hline Urokinase & $1 \mathrm{C} 5 \mathrm{X}$ & 4DVA & 246 & 0.429 & ESI \\
\hline Urokinase & $1 \mathrm{C} 5 \mathrm{Y}$ & 4DVA & 246 & 0.43 & ESP \\
\hline Urokinase & $1 \mathrm{C} 5 \mathrm{Z}$ & 4DVA & 246 & 0.44 & BAM \\
\hline Urokinase & $1 \mathrm{EJN}$ & 4DVA & 246 & 0.404 & AGB \\
\hline Urokinase & $1 \mathrm{~F} 5 \mathrm{~K}$ & 4DVA & 246 & 0.377 & BAM \\
\hline Urokinase & $1 \mathrm{~F} 5 \mathrm{~L}$ & 4DVA & 246 & 0.379 & AMR \\
\hline Urokinase & $1 \mathrm{~F} 92$ & 4DVA & 246 & 0.397 & UKP \\
\hline Urokinase & $1 \mathrm{GI} 7$ & 4DVA & 246 & 0.446 & 120 \\
\hline Urokinase & $1 \mathrm{GI} 8$ & 4DVA & 246 & 0.429 & BMZ \\
\hline Urokinase & $1 \mathrm{GI} 9$ & 4DVA & 246 & 0.43 & 123 \\
\hline Urokinase & 1GJ7 & 4DVA & 246 & 0.409 & 132 \\
\hline Urokinase & 1GJ8 & 4DVA & 246 & 0.416 & 133 \\
\hline Urokinase & $1 \mathrm{GJA}$ & 4DVA & 246 & 0.396 & 135 \\
\hline
\end{tabular}




\begin{tabular}{|c|c|c|c|c|c|}
\hline Urokinase & $1 \mathrm{GJB}$ & 4DVA & 246 & 0.465 & 130 \\
\hline Urokinase & $1 \mathrm{GJC}$ & 4DVA & 246 & 0.431 & 130 \\
\hline Urokinase & $1 \mathrm{GJD}$ & 4DVA & 246 & 0.405 & 136 \\
\hline Urokinase & $1 \mathrm{O} 3 \mathrm{P}$ & 4DVA & 246 & 0.438 & 655 \\
\hline Urokinase & $1 \mathrm{O} 5 \mathrm{~A}$ & 4DVA & 246 & 0.416 & 696 \\
\hline Urokinase & $1 \mathrm{O} 5 \mathrm{~B}$ & 4DVA & 246 & 0.42 & ESI \\
\hline Urokinase & $1 \mathrm{O5C}$ & 4DVA & 246 & 0.408 & CR9 \\
\hline
\end{tabular}

* Template PDB ids used for modeling unbound structures.

Table S2. Pearson Correlation coefficients between the energy scores obtained from RosettaLigand bound, RosettaLigand unbound and Adaptive BP-Dock unbound docking and the experimental binding affinities for 5 individual runs for HIV-1 Protease (PR) and Carbonic Anhydrase II (CAII) for three different iteration parameters of 5,10 and 20.

\begin{tabular}{|c|c|c|c|c|c|c|c|c|}
\hline \multirow[b]{2}{*}{$\begin{array}{l}\text { Iteration } \\
\text { Parameter }\end{array}$} & \multirow[b]{2}{*}{ Protein } & \multirow[b]{2}{*}{ Run \# } & \multicolumn{3}{|c|}{ RosettaLigand Score (kcal/mol) } & \multicolumn{3}{|c|}{$\mathrm{X}$-Score $(\mathrm{kcal} / \mathrm{mol})$} \\
\hline & & & Bound & Unbound & $\begin{array}{c}\text { ABP- } \\
\text { Unbound }\end{array}$ & Bound & Unbound & $\begin{array}{c}\text { ABP- } \\
\text { Unbound }\end{array}$ \\
\hline \multirow{12}{*}{5} & \multirow{6}{*}{ PR } & 1run & 0.84 & 0.69 & 0.86 & 0.83 & 0.78 & 0.89 \\
\hline & & 2run & 0.82 & 0.70 & 0.86 & 0.83 & 0.76 & 0.88 \\
\hline & & 3run & 0.84 & 0.67 & 0.88 & 0.83 & 0.79 & 0.87 \\
\hline & & 4run & 0.81 & 0.66 & 0.84 & 0.84 & 0.77 & 0.88 \\
\hline & & 5run & 0.83 & 0.67 & 0.86 & 0.83 & 0.76 & 0.89 \\
\hline & & AVERAGE & 0.83 & 0.68 & 0.86 & 0.83 & 0.77 & 0.88 \\
\hline & \multirow{6}{*}{ CA II } & 1run & 0.87 & 0.71 & 0.75 & 0.69 & 0.68 & 0.91 \\
\hline & & 2run & 0.87 & 0.70 & 0.76 & 0.68 & 0.66 & 0.92 \\
\hline & & 3run & 0.86 & 0.69 & 0.76 & 0.67 & 0.65 & 0.91 \\
\hline & & 4run & 0.88 & 0.71 & 0.75 & 0.68 & 0.67 & 0.90 \\
\hline & & 5run & 0.87 & 0.70 & 0.75 & 0.69 & 0.68 & 0.91 \\
\hline & & AVERAGE & 0.87 & 0.70 & 0.75 & 0.68 & 0.67 & 0.91 \\
\hline \multirow{12}{*}{10} & \multirow{6}{*}{ PR } & 1run & 0.76 & 0.61 & 0.79 & 0.81 & 0.72 & 0.78 \\
\hline & & 2run & 0.73 & 0.62 & 0.78 & 0.79 & 0.74 & 0.76 \\
\hline & & 3run & 0.75 & 0.61 & 0.77 & 0.78 & 0.68 & 0.75 \\
\hline & & 4run & 0.75 & 0.64 & 0.78 & 0.80 & 0.69 & 0.76 \\
\hline & & 5run & 0.76 & 0.60 & 0.77 & 0.81 & 0.73 & 0.77 \\
\hline & & AVERAGE & 0.75 & 0.62 & 0.78 & 0.80 & 0.71 & 0.76 \\
\hline & \multirow{6}{*}{ CA II } & 1run & 0.81 & 0.64 & 0.69 & 0.55 & 0.56 & 0.85 \\
\hline & & 2run & 0.80 & 0.63 & 0.69 & 0.55 & 0.54 & 0.85 \\
\hline & & 3run & 0.81 & 0.62 & 0.67 & 0.56 & 0.55 & 0.86 \\
\hline & & 4run & 0.80 & 0.64 & 0.70 & 0.57 & 0.58 & 0.85 \\
\hline & & 5run & 0.79 & 0.62 & 0.68 & 0.56 & 0.57 & 0.85 \\
\hline & & AVERAGE & 0.80 & 0.63 & 0.69 & 0.56 & 0.56 & 0.85 \\
\hline \multirow[b]{3}{*}{20} & \multirow{3}{*}{ PR } & 1run & 0.86 & 0.70 & 0.86 & 0.84 & 0.78 & 0.90 \\
\hline & & 2run & 0.84 & 0.69 & 0.88 & 0.84 & 0.79 & 0.89 \\
\hline & & 3run & 0.85 & 0.69 & 0.87 & 0.85 & 0.79 & 0.88 \\
\hline
\end{tabular}




\begin{tabular}{|c|c|c|c|c|c|c|c|}
\hline & 4run & 0.84 & 0.71 & 0.85 & 0.85 & 0.78 & 0.89 \\
\hline & 5run & 0.86 & 0.71 & 0.86 & 0.84 & 0.80 & 0.91 \\
\hline & AVERAGE & 0.85 & 0.70 & 0.86 & 0.84 & 0.79 & 0.89 \\
\hline \multirow{6}{*}{ CA II } & 1run & 0.88 & 0.74 & 0.78 & 0.71 & 0.69 & 0.91 \\
\hline & 2 run & 0.87 & 0.74 & 0.79 & 0.74 & 0.68 & 0.91 \\
\hline & 3 run & 0.89 & 0.75 & 0.79 & 0.73 & 0.69 & 0.92 \\
\hline & 4run & 0.88 & 0.73 & 0.77 & 0.70 & 0.69 & 0.91 \\
\hline & 5run & 0.87 & 0.75 & 0.78 & 0.73 & 0.70 & 0.91 \\
\hline & AVERAGE & 0.88 & 0.74 & 0.78 & 0.72 & 0.69 & 0.91 \\
\hline
\end{tabular}

Table S3. RosettaLigand energy scores and X-Scores for the unbound docking of HIV-1 Protease and Alcohol Dehydrogenase for two different iteration parameters.

\begin{tabular}{|c|c|c|c|c|c|c|c|}
\hline \multirow[b]{2}{*}{ Protein } & \multicolumn{2}{|c|}{ PDB Code } & \multicolumn{2}{|c|}{$\begin{array}{c}1 \text { Iteration } \\
\text { (10000 snapshots/iteration) }\end{array}$} & \multicolumn{2}{|c|}{$\begin{array}{c}10 \text { Iterations } \\
\text { (1000 snapshots/iteration) }\end{array}$} & \multirow{2}{*}{$\operatorname{Exp} \Delta \mathbf{G}$} \\
\hline & Bound & Unbound & $\begin{array}{c}\text { RosettaLigand } \\
\text { Score } \\
(\text { kcal } / \mathbf{m o l})\end{array}$ & $\begin{array}{c}\text { X-Score } \\
(\text { kcal} / \mathbf{m o l})\end{array}$ & $\begin{array}{c}\text { RosettaLigand } \\
\text { Score } \\
(\text { kcal } / \mathbf{m o l})\end{array}$ & $\begin{array}{c}\text { X-Score } \\
(\text { kcal/mol) }\end{array}$ & \\
\hline \multirow{21}{*}{ HIV Protease } & $1 \mathrm{HBV}$ & $2 \mathrm{PC} 0$ & -10.45 & -8.93 & -10.38 & -8.92 & -8.68 \\
\hline & 1HEG & $2 \mathrm{PC} 0$ & -10.77 & -8.71 & -10.82 & -8.73 & -10.38 \\
\hline & $1 \mathrm{HIH}$ & $2 \mathrm{PC} 0$ & -13.18 & -9.68 & -13.38 & -9.68 & -10.97 \\
\hline & $1 \mathrm{HIV}$ & $2 \mathrm{PCO}$ & -18.76 & -10.11 & -18.65 & -10.09 & -12.64 \\
\hline & 1HPS & $2 \mathrm{PC} 0$ & -18.19 & -10 & -18.25 & -9.95 & -12.66 \\
\hline & 1HTE & $2 \mathrm{PC} 0$ & -10.64 & -8.47 & -10.54 & -8.47 & -7.69 \\
\hline & 1HTF & $2 \mathrm{PCO}$ & -11.33 & -8.64 & -11.39 & -8.63 & -11.04 \\
\hline & 1HTG & $2 \mathrm{PC} 0$ & -19.99 & -10.56 & -20.26 & -10.57 & -13.2 \\
\hline & 1HVI & $2 \mathrm{PCO}$ & -18.64 & -10.16 & -18.62 & -10.18 & -13.74 \\
\hline & $1 \mathrm{HVJ}$ & $2 \mathrm{PC} 0$ & -18.35 & -10.27 & -18.22 & -10.27 & -14.26 \\
\hline & 1HVK & $2 \mathrm{PCO}$ & -18.79 & -10.36 & -18.56 & -10.35 & -13.79 \\
\hline & 1HVL & $2 \mathrm{PC} 0$ & -17.75 & -10.01 & -17.48 & -10.04 & -12.27 \\
\hline & 1HVS & $2 \mathrm{PCO}$ & -18.08 & -9.06 & -18.19 & -9.08 & -13.81 \\
\hline & $1 \mathrm{SBG}$ & $2 \mathrm{PC} 0$ & -16.19 & -9.55 & -16.38 & -9.5 & -10.38 \\
\hline & 4HVP & $2 \mathrm{PCO}$ & -18.68 & -9.6 & -18.45 & -9.58 & -8.33 \\
\hline & 4PHV & $2 \mathrm{PCO}$ & -23.87 & -11.28 & -23.19 & -11.29 & -12.56 \\
\hline & $5 \mathrm{HVP}$ & $2 \mathrm{PCO}$ & -11.91 & -8.75 & -11.14 & -8.75 & -10.5 \\
\hline & 9HVP & $2 \mathrm{PCO}$ & -15.13 & -9.64 & -14.99 & -9.61 & -11.38 \\
\hline & $1 \mathrm{~A} 30$ & $2 \mathrm{PCO}$ & -9.68 & -6.68 & -9.62 & -6.64 & -5.77 \\
\hline & $1 \mathrm{KZK}$ & $2 \mathrm{PCO}$ & -16.13 & -10.12 & -16.38 & -10.15 & -13.94 \\
\hline & \multicolumn{2}{|c|}{ Correlation } & 0.692636812 & 0.778623941 & 0.705424511 & 0.783548874 & \\
\hline \multirow{6}{*}{$\begin{array}{c}\text { Alcohol } \\
\text { Dehydrogenase }\end{array}$} & 1ADB & $8 \mathrm{ADH}$ & -18.93 & -9.05 & -19.02 & -9.09 & -11.45 \\
\hline & $1 \mathrm{ADC}$ & $8 \mathrm{ADH}$ & -18.81 & -9.11 & -18.95 & -9.12 & -6.42 \\
\hline & $1 \mathrm{ADF}$ & $8 \mathrm{ADH}$ & -16.75 & -8.59 & -16.64 & -8.55 & -6.24 \\
\hline & 1BTO & $8 \mathrm{ADH}$ & -8.83 & -6.45 & -8.96 & -6.47 & -8.93 \\
\hline & 1HLD & $8 \mathrm{ADH}$ & -6.19 & -5.99 & -6.01 & -5.98 & -7.58 \\
\hline & $1 \mathrm{LDE}$ & $8 \mathrm{ADH}$ & -6.94 & -6.02 & -7.01 & -6.07 & -9.41 \\
\hline
\end{tabular}




\begin{tabular}{|c|c|c|c|c|c|c|c|}
\hline \multirow{2}{*}{$1 \mathrm{LDY}$} & $8 \mathrm{ADH}$ & -6.76 & -6.05 & -6.75 & -6.02 & -11.06 \\
\cline { 2 - 8 } & $3 \mathrm{BTO}$ & $8 \mathrm{ADH}$ & -8.98 & -6.48 & -8.86 & -6.47 & -8.43 \\
\cline { 2 - 8 } & \multicolumn{2}{|c|}{ Correlation } & -0.230382554 & -0.25111957 & -0.222157341 & -0.24203658 & \\
\hline
\end{tabular}

Table S4. The ligand RMSD $(\AA)$ values between the ligand positions of the lowest energy docked poses obtained from RosettaLigand bound, RosettaLigand unbound and Adaptive BP-Dock unbound docking to that of the native ligand (Only heavy atoms of the ligand are taken into account for RMSD calculation).

\begin{tabular}{|c|c|c|c|c|}
\hline \multirow{4}{*}{ Protein } & \multirow{4}{*}{ Peptide/Ligand } & \multicolumn{3}{|c|}{$\mathbf{R M S D}_{\text {ligand }}(\AA)$} \\
\hline & & \multicolumn{2}{|c|}{ RosettaLigand Flexible } & \multirow{2}{*}{$\begin{array}{c}\text { Adaptive BP-Dock } \\
\text { Unbound }\end{array}$} \\
\hline & & Bound & Unbound & \\
\hline & & (Holo) & (Apo) & (Apo) \\
\hline HIV-Protease & SB203238 & 0.418003 & 1.2791 & 1.08985 \\
\hline HIV-Protease & SKF 107457 & 0.709186 & 1.56862 & 1.39926 \\
\hline HIV-Protease & CGP-53820 & 0.235182 & 0.775262 & 1.22041 \\
\hline HIV-Protease & U75875 & 0.193206 & 0.866439 & 0.60067 \\
\hline HIV-Protease & SB206343 & 0.175098 & 0.973543 & 0.42924 \\
\hline HIV-Protease & GR123976 & 0.638352 & 0.848704 & 0.861571 \\
\hline HIV-Protease & GR126045 & 0.531159 & 1.41543 & 1.27397 \\
\hline HIV-Protease & GR137615 & 0.37927 & 1.45318 & 0.941731 \\
\hline HIV-Protease & A77003 (R,S) & 0.222304 & 2.06155 & 1.03451 \\
\hline HIV-Protease & A78791 (S,-) & 0.225837 & 2.07783 & 1.69103 \\
\hline HIV-Protease & A76928 (S,S) & 0.191069 & 2.02997 & 1.48351 \\
\hline HIV-Protease & A76889 (R,R) & 0.456418 & 2.1789 & 1.29947 \\
\hline HIV-Protease & A77 & 0.350743 & 2.21229 & 1.22437 \\
\hline HIV-Protease & IM1 & 0.495815 & 0.971637 & 0.896572 \\
\hline HIV-Protease & MVT-101 & 0.54874 & 0.954794 & 0.84661 \\
\hline HIV-Protease & L-700, 417 & 0.308484 & 0.718445 & 0.995701 \\
\hline HIV-Protease & $\begin{array}{c}\text { ACETYL- } \\
\text { PEPSTATIN }\end{array}$ & 0.423483 & 1.75529 & 1.21663 \\
\hline HIV-Protease & A-74704 & 0.366486 & 2.88295 & 1.97908 \\
\hline HIV-Protease & Tri-peptide & 4.77909 & 1.23006 & 1.37058 \\
\hline HIV-Protease & JE-2147 & 0.253889 & 0.779713 & 0.74522 \\
\hline Carbonic Anhydrase II & Celecoxib & 0.67636 & 1.75736 & 0.40954 \\
\hline Carbonic Anhydrase II & Histamine & 0.310267 & 0.268871 & 0.231485 \\
\hline Carbonic Anhydrase II & ETS & 0.365087 & 0.396402 & 0.574027 \\
\hline Carbonic Anhydrase II & PTS & 0.283476 & 0.634483 & 0.341912 \\
\hline Carbonic Anhydrase II & MTS & 0.974404 & 0.587369 & 0.569293 \\
\hline Carbonic Anhydrase II & EG1 & 0.498755 & 0.608803 & 0.449081 \\
\hline
\end{tabular}




\begin{tabular}{|c|c|c|c|c|}
\hline Carbonic Anhydrase II & EG2 & 0.608116 & 0.862314 & 0.726957 \\
\hline Carbonic Anhydrase II & EG3 & 0.563141 & 0.708502 & 0.583964 \\
\hline Carbonic Anhydrase II & MNS & 0.413472 & 0.672734 & 0.549698 \\
\hline Alcohol Deydrogenase & CND & 0.175106 & 1.08212 & 0.98551 \\
\hline Alcohol Deydrogenase & PAD & 0.356442 & 1.36048 & 1.22878 \\
\hline Alcohol Deydrogenase & TAD & 0.459939 & 1.21281 & 1.22144 \\
\hline Alcohol Deydrogenase & SSB & 0.587668 & 1.3735 & 1.14636 \\
\hline Alcohol Deydrogenase & $\mathrm{PFB}, \mathrm{NAD}$ & 0.992785 & 1.41033 & 1.13083 \\
\hline Alcohol Deydrogenase & FPI,NAD & 0.282478 & 1.43041 & 0.913254 \\
\hline Alcohol Deydrogenase & CXF,NAD & 1.25703 & 1.71723 & 1.735 \\
\hline Alcohol Deydrogenase & $\mathrm{SSB}, \mathrm{NAD}$ & 1.82204 & 1.04608 & 0.579008 \\
\hline Alpha-Thrombin & QWE & 0.403345 & 0.664845 & 0.575657 \\
\hline Alpha-Thrombin & AZL & 0.267047 & 0.433209 & 0.317985 \\
\hline Alpha-Thrombin & BMS-186282 & 0.582128 & 0.503689 & 0.40902 \\
\hline Alpha-Thrombin & BMS-189090 & 0.383565 & 1.63934 & 0.763905 \\
\hline Alpha-Thrombin & BZT & 0.330468 & 0.59422 & 0.520805 \\
\hline Alpha-Thrombin & ВT3 & 0.299717 & 0.291204 & 0.384348 \\
\hline Alpha-Thrombin & BPP & 0.369666 & 0.440537 & 0.412269 \\
\hline Alpha-Thrombin & Benzyldiamine & 0.447416 & 0.573638 & 0.675049 \\
\hline Alpha-Thrombin & $\begin{array}{c}\text { MD-805 } \\
\text { (Argatroban) } \\
\end{array}$ & 0.405094 & 0.562457 & 0.490317 \\
\hline Alpha-Thrombin & NAPAP & 0.401877 & 0.609482 & 0.414369 \\
\hline Alpha-Thrombin & BMS-183507 & 0.511108 & 1.23994 & 0.951574 \\
\hline Alpha-Thrombin & BM51.1011 & 0.808384 & 0.689386 & 0.65194 \\
\hline Alpha-Thrombin & FSN & 0.312475 & 1.42675 & 0.684689 \\
\hline Cytohchrome C Peroxidase & TMT & 0.777135 & 0.839883 & 0.82789 \\
\hline Cytohchrome C Peroxidase & TMZ & 0.621377 & 0.88462 & 0.83291 \\
\hline Cytohchrome C Peroxidase & $3 \mathrm{MT}$ & 0.46717 & 0.228232 & 0.38842 \\
\hline Cytohchrome C Peroxidase & DTI & 0.6142 & 0.656081 & 0.897984 \\
\hline Cytohchrome C Peroxidase & ANL & 0.480588 & 0.396096 & 0.542739 \\
\hline Cytohchrome C Peroxidase & $3 \mathrm{AP}$ & 0.708662 & 1.30767 & 0.724415 \\
\hline Cytohchrome C Peroxidase & $4 \mathrm{AP}$ & 0.810646 & 1.43459 & 1.08697 \\
\hline Cytohchrome C Peroxidase & $24 \mathrm{~T}$ & 0.7854 & 0.688599 & 0.819344 \\
\hline Cytohchrome C Peroxidase & NVI & 0.790638 & 0.70824 & 0.972393 \\
\hline Cytohchrome C Peroxidase & IDM & 0.929259 & 1.20286 & 1.07223 \\
\hline Cytohchrome C Peroxidase & MPI & 0.683565 & 1.15626 & 1.25543 \\
\hline Cytohchrome C Peroxidase & $25 \mathrm{~T}$ & 0.656364 & 0.631935 & 0.788862 \\
\hline Cytohchrome C Peroxidase & $2 \mathrm{AP}$ & 0.608017 & 0.548956 & 0.689105 \\
\hline Cytohchrome C Peroxidase & $2 \mathrm{EZ}$ & 0.376223 & 0.410639 & 0.74565 \\
\hline Cytohchrome C Peroxidase & IMD & 0.589494 & 0.625658 & 0.528747 \\
\hline Cytohchrome C Peroxidase & $1 \mathrm{MZ}$ & 0.825651 & 0.197187 & 0.69063 \\
\hline
\end{tabular}




\begin{tabular}{|c|c|c|c|c|}
\hline Cytohchrome C Peroxidase & $2 \mathrm{MZ}$ & 0.765318 & 1.57498 & 0.937405 \\
\hline Cytohchrome C Peroxidase & AMT & 0.372296 & 0.759523 & 0.802553 \\
\hline HIV-1 Reverse Transcriptase & FPT & 0.247778 & 2.51296 & 2.25609 \\
\hline HIV-1 Reverse Transcriptase & FTC & 0.420908 & 2.88472 & 1.41058 \\
\hline HIV-1 Reverse Transcriptase & EFZ & 0.637945 & 2.83467 & 1.80847 \\
\hline HIV-1 Reverse Transcriptase & EFZ & 0.815516 & 2.54711 & 1.44091 \\
\hline HIV-1 Reverse Transcriptase & PNU & 0.227793 & 3.76825 & 3.00962 \\
\hline HIV-1 Reverse Transcriptase & MSD & 0.194144 & 4.10376 & 2.50698 \\
\hline HIV-1 Reverse Transcriptase & SBN & 0.348824 & 5.2524 & 2.51296 \\
\hline HIV-1 Reverse Transcriptase & $\mathrm{H} 12$ & 0.299607 & 6.77265 & 2.65651 \\
\hline HIV-1 Reverse Transcriptase & GWB & 0.431185 & 6.96777 & 2.60713 \\
\hline HIV-1 Reverse Transcriptase & H16 & 0.349549 & 5.47772 & 1.83542 \\
\hline HIV-1 Reverse Transcriptase & $\mathrm{H} 18$ & 0.526607 & 6.48027 & 2.28445 \\
\hline HIV-1 Reverse Transcriptase & $\mathrm{H} 20$ & 0.335853 & 3.46953 & 2.12041 \\
\hline HIV-1 Reverse Transcriptase & $\mathrm{CP} 9$ & 1.21715 & 3.60338 & 3.92662 \\
\hline HIV-1 Reverse Transcriptase & $3 \mathrm{AC}$ & 0.20557 & 3.29358 & 2.61775 \\
\hline HIV-1 Reverse Transcriptase & 357 & 0.623558 & 4.85703 & 2.58896 \\
\hline HIV-1 Reverse Transcriptase & $\mathrm{R} 22$ & 0.229452 & 6.36034 & 3.39196 \\
\hline HIV-1 Reverse Transcriptase & NVP & 0.300807 & 3.73621 & 3.08572 \\
\hline HIV-1 Reverse Transcriptase & RT3 & 0.242092 & 2.21197 & 2.85327 \\
\hline HIV-1 Reverse Transcriptase & RT7 & 0.423012 & 2.65632 & 2.23095 \\
\hline HIV-1 Reverse Transcriptase & UDR & 0.378177 & 4.2607 & 2.41051 \\
\hline HIV-1 Reverse Transcriptase & AC7 & 0.71602 & 3.2904 & 3.23178 \\
\hline HIV-1 Reverse Transcriptase & LP9 & 30.4517 & 3.2138 & 3.40637 \\
\hline HIV-1 Reverse Transcriptase & $65 \mathrm{~B}$ & 0.26778 & 2.78793 & 2.2235 \\
\hline HIV-1 Reverse Transcriptase & DJZ & 0.187866 & 2.14591 & 2.2416 \\
\hline HIV-1 Reverse Transcriptase & QO9 & 0.429494 & 1.5736 & 1.23462 \\
\hline Urokinase & ESI & 0.274541 & 0.40308 & 0.457713 \\
\hline Urokinase & ESP & 0.264107 & 0.275177 & 0.235237 \\
\hline Urokinase & BAM & 0.331864 & 0.340833 & 0.325066 \\
\hline Urokinase & AGB & 0.222822 & 0.825686 & 0.728388 \\
\hline Urokinase & BAM & 0.27238 & 0.571728 & 0.34185 \\
\hline Urokinase & AMR & 0.450419 & 0.600509 & 0.520076 \\
\hline Urokinase & UKP & 0.394323 & 1.84406 & 1.32854 \\
\hline Urokinase & 120 & 1.06065 & 0.745108 & 0.981233 \\
\hline Urokinase & $\mathrm{BMZ}$ & 0.385946 & 0.726855 & 0.914602 \\
\hline
\end{tabular}




\begin{tabular}{|c|c|c|c|c|}
\hline Urokinase & 123 & 0.489032 & 1.00727 & 0.734616 \\
\hline Urokinase & 132 & 0.425829 & 0.343307 & 0.552314 \\
\hline Urokinase & 133 & 0.228622 & 0.860835 & 1.29 \\
\hline Urokinase & 135 & 0.205307 & 0.483332 & 0.888727 \\
\hline Urokinase & 130 & 0.345033 & 0.53488 & 0.910093 \\
\hline Urokinase & 130 & 0.580323 & 0.564825 & 1.21495 \\
\hline Urokinase & 136 & 0.382296 & 0.317818 & 0.828407 \\
\hline Urokinase & 655 & 0.333867 & 0.416087 & 0.787138 \\
\hline Urokinase & 696 & 1.04054 & 1.08315 & 1.15058 \\
\hline Urokinase & ESI & 0.34186 & 0.193498 & 0.326192 \\
\hline Urokinase & CR9 & 1.14861 & 0.395891 & 0.45604 \\
\hline
\end{tabular}

Table S5. All-atom and backbone RMSD ( $\AA$ ) between the Bound and (a) Unbound experimental structures of the proteins, (b) the best docked pose obtained from 'flexiblebackbone' protocol docking of unbound protein with RosettaLigand, (c) the best-docked pose obtained from 'Adaptive BP-Dock' docking of unbound protein.

\begin{tabular}{|c|c|c|c|c|c|c|c|}
\hline \multirow[b]{2}{*}{ Protein } & \multirow[b]{2}{*}{$\begin{array}{l}\text { Peptide/ } \\
\text { Ligand }\end{array}$} & \multicolumn{3}{|c|}{$\mathbf{R M S D}_{\text {all-atom }}(\mathbf{A})$} & \multicolumn{3}{|c|}{$\mathbf{R M S D}_{\text {backbone }}(\AA)$} \\
\hline & & $\begin{array}{l}\text { Bound- } \\
\text { Unbound }\end{array}$ & $\begin{array}{c}\text { Bound- } \\
\text { RosettaLiga } \\
\text { nd Unbound } \\
\text { Docked } \\
\text { Pose }\end{array}$ & $\begin{array}{c}\text { Bound- } \\
\text { Adaptive } \\
\text { BP-Dock } \\
\text { Unbound } \\
\text { Docked } \\
\text { Pose } \\
\end{array}$ & $\begin{array}{l}\text { Bound- } \\
\text { Unbound }\end{array}$ & $\begin{array}{c}\text { Bound- } \\
\text { RosettaLiga } \\
\text { nd Unbound } \\
\text { Docked } \\
\text { Pose }\end{array}$ & $\begin{array}{c}\text { Bound- } \\
\text { Adaptive BP- } \\
\text { Dock } \\
\text { Unbound } \\
\text { Docked Pose }\end{array}$ \\
\hline HIV-Protease & SB203238 & 1.65 & 1.641 & 1.263 & 1.461 & 1.347 & 1.102 \\
\hline HIV-Protease & SKF 107457 & 1.614 & 1.618 & 1.081 & 1.517 & 1.542 & 0.893 \\
\hline HIV-Protease & CGP-53820 & 1.343 & 1.328 & 1.324 & 1.17 & 1.287 & 1.124 \\
\hline HIV-Protease & U75875 & 1.422 & 1.405 & 1.206 & 1.191 & 1.238 & 1.082 \\
\hline HIV-Protease & SB206343 & 1.606 & 1.603 & 1.013 & 1.4 & 1.458 & 0.891 \\
\hline HIV-Protease & GR123976 & 1.505 & 1.519 & 1.504 & 1.395 & 1.456 & 1.349 \\
\hline HIV-Protease & GR126045 & 1.436 & 1.447 & 0.921 & 1.327 & 1.231 & 0.782 \\
\hline HIV-Protease & GR137615 & 1.405 & 1.399 & 1.129 & 1.343 & 1.298 & 1.067 \\
\hline HIV-Protease & $\begin{array}{c}\text { A77003 } \\
(\mathrm{R}, \mathrm{S})\end{array}$ & 1.344 & 1.352 & 1.214 & 1.222 & 1.091 & 1.002 \\
\hline HIV-Protease & A78791 (S,-) & 1.347 & 1.341 & 1.229 & 1.231 & 1.295 & 1.117 \\
\hline HIV-Protease & $\begin{array}{c}\text { A76928 } \\
(\mathrm{S}, \mathrm{S}) \\
\end{array}$ & 1.352 & 1.349 & 1.028 & 1.247 & 1.194 & 0.993 \\
\hline HIV-Protease & $\begin{array}{c}\text { A76889 } \\
(\mathrm{R}, \mathrm{R})\end{array}$ & 1.347 & 1.363 & 1.449 & 1.251 & 1.314 & 1.183 \\
\hline HIV-Protease & A77 & 1.406 & 1.414 & 1.194 & 1.265 & 1.301 & 1.148 \\
\hline HIV-Protease & IM1 & 1.561 & 1.571 & 1.185 & 1.389 & 1.472 & 0.993 \\
\hline HIV-Protease & MVT-101 & 1.538 & 1.536 & 1.214 & 1.337 & 1.225 & 1.114 \\
\hline HIV-Protease & L-700, 417 & 1.373 & 1.356 & 1.392 & 1.302 & 1.278 & 1.131 \\
\hline
\end{tabular}




\begin{tabular}{|c|c|c|c|c|c|c|c|}
\hline HIV-Protease & $\begin{array}{c}\text { ACETYL- } \\
\text { PEPSTATIN }\end{array}$ & 1.427 & 1.421 & 1.128 & 1.386 & 1.225 & 1.021 \\
\hline HIV-Protease & A-74704 & 1.512 & 1.522 & 1.184 & 1.324 & 1.431 & 0.999 \\
\hline HIV-Protease & Tri-peptide & 1.49 & 1.406 & 1.199 & 1.438 & 1.235 & 1.024 \\
\hline HIV-Protease & JE-2147 & 1.253 & 1.237 & 1.318 & 1.127 & 1.116 & 1.215 \\
\hline $\begin{array}{c}\text { Carbonic } \\
\text { Anhydrase II }\end{array}$ & Celecoxib & 0.134 & 0.132 & 0.112 & 0.122 & 0.118 & 0.094 \\
\hline $\begin{array}{c}\text { Carbonic } \\
\text { Anhydrase II }\end{array}$ & Histamine & 0.254 & 0.233 & 0.194 & 0.232 & 0.21 & 0.174 \\
\hline $\begin{array}{c}\text { Carbonic } \\
\text { Anhydrase II }\end{array}$ & ETS & 0.239 & 0.28 & 0.212 & 0.224 & 0.271 & 0.194 \\
\hline $\begin{array}{c}\text { Carbonic } \\
\text { Anhydrase II }\end{array}$ & PTS & 0.258 & 0.241 & 0.238 & 0.218 & 0.236 & 0.212 \\
\hline $\begin{array}{c}\text { Carbonic } \\
\text { Anhydrase II }\end{array}$ & MTS & 0.244 & 0.25 & 0.251 & 0.212 & 0.229 & 0.217 \\
\hline $\begin{array}{c}\text { Carbonic } \\
\text { Anhydrase II }\end{array}$ & EG1 & 0.231 & 0.228 & 0.217 & 0.212 & 0.201 & 0.198 \\
\hline $\begin{array}{c}\text { Carbonic } \\
\text { Anhydrase II }\end{array}$ & EG2 & 0.248 & 0.256 & 0.204 & 0.227 & 0.216 & 0.183 \\
\hline $\begin{array}{c}\text { Carbonic } \\
\text { Anhydrase II }\end{array}$ & EG3 & 0.258 & 0.27 & 0.261 & 0.234 & 0.195 & 0.179 \\
\hline $\begin{array}{c}\text { Carbonic } \\
\text { Anhydrase II }\end{array}$ & MNS & 0.308 & 0.216 & 0.204 & 0.298 & 0.193 & 0.177 \\
\hline $\begin{array}{c}\text { Alcohol } \\
\text { Deydrogenase }\end{array}$ & CND & 1.218 & 1.201 & 1.229 & 1.107 & 1.102 & 1.004 \\
\hline $\begin{array}{c}\text { Alcohol } \\
\text { Deydrogenase }\end{array}$ & PAD & 1.219 & 2.214 & 1.118 & 1.111 & 0.891 & 0.999 \\
\hline $\begin{array}{c}\text { Alcohol } \\
\text { Deydrogenase }\end{array}$ & TAD & 0.33 & 0.332 & 0.491 & 0.284 & 0.226 & 0.375 \\
\hline $\begin{array}{c}\text { Alcohol } \\
\text { Deydrogenase }\end{array}$ & SSB & 1.137 & 1.126 & 0.984 & 1.043 & 0.958 & 0.824 \\
\hline $\begin{array}{c}\text { Alcohol } \\
\text { Deydrogenase }\end{array}$ & $\mathrm{PFB}, \mathrm{NAD}$ & 1.135 & 1.148 & 1.119 & 1.033 & 1.123 & 1.017 \\
\hline $\begin{array}{c}\text { Alcohol } \\
\text { Deydrogenase }\end{array}$ & FPI,NAD & 1.148 & 1.136 & 1.129 & 1.029 & 1.045 & 1.002 \\
\hline $\begin{array}{c}\text { Alcohol } \\
\text { Deydrogenase }\end{array}$ & CXF,NAD & 1.152 & 1.146 & 1.102 & 1.017 & 1.037 & 0.994 \\
\hline $\begin{array}{c}\text { Alcohol } \\
\text { Deydrogenase } \\
\end{array}$ & $\mathrm{SSB}, \mathrm{NAD}$ & 1.16 & 1.15 & 1.083 & 1.051 & 1.003 & 1.01 \\
\hline Alpha-Thrombin & QWE & 0.351 & 0.336 & 0.282 & 0.307 & 0.289 & 0.226 \\
\hline Alpha-Thrombin & AZL & 0.331 & 0.215 & 0.284 & 0.179 & 0.193 & 0.184 \\
\hline Alpha-Thrombin & $\begin{array}{c}\text { BMS- } \\
186282 \\
\end{array}$ & 0.455 & 0.461 & 0.371 & 0.409 & 0.319 & 0.367 \\
\hline Alpha-Thrombin & $\begin{array}{l}\text { BMS- } \\
189090\end{array}$ & 0.423 & 0.413 & 0.392 & 0.373 & 0.323 & 0.341 \\
\hline Alpha-Thrombin & BZT & 0.352 & 0.356 & 0.319 & 0.319 & 0.319 & 0.292 \\
\hline Alpha-Thrombin & BT3 & 0.317 & 0.318 & 0.301 & 0.272 & 0.286 & 0.273 \\
\hline Alpha-Thrombin & BPP & 0.333 & 0.333 & 0.308 & 0.303 & 0.292 & 0.217 \\
\hline Alpha-Thrombin & $\begin{array}{l}\text { Benzyldiami } \\
\text { ne }\end{array}$ & 0.386 & 0.291 & 0.341 & 0.243 & 0.225 & 0.278 \\
\hline Alpha-Thrombin & $\begin{array}{c}\text { MD-805 } \\
\text { (Argatroban) }\end{array}$ & 0.386 & 0.28 & 0.329 & 0.235 & 0.193 & 0.226 \\
\hline Alpha-Thrombin & NAPAP & 0.378 & 0.291 & 0.331 & 0.238 & 0.208 & 0.238 \\
\hline Alpha-Thrombin & $\begin{array}{c}\text { BMS- } \\
183507 \\
\end{array}$ & 0.511 & 0.513 & 0.482 & 0.429 & 0.441 & 0.385 \\
\hline Alpha-Thrombin & BM51.1011 & 0.479 & 0.466 & 0.481 & 0.392 & 0.295 & 0.222 \\
\hline Alpha-Thrombin & FSN & 0.387 & 0.389 & 0.392 & 0.359 & 0.347 & 0.291 \\
\hline
\end{tabular}




\begin{tabular}{|c|c|c|c|c|c|c|c|}
\hline $\begin{array}{c}\text { Cytohchrome } \mathrm{C} \\
\text { Peroxidase }\end{array}$ & TMT & 0.286 & 0.284 & 0.292 & 0.253 & 0.227 & 0.218 \\
\hline $\begin{array}{c}\text { Cytohchrome } \mathrm{C} \\
\text { Peroxidase }\end{array}$ & TMZ & 0.299 & 0.301 & 0.278 & 0.276 & 0.209 & 0.226 \\
\hline $\begin{array}{c}\text { Cytohchrome } \mathrm{C} \\
\text { Peroxidase }\end{array}$ & $3 \mathrm{MT}$ & 0.299 & 0.299 & 0.301 & 0.275 & 0.278 & 0.295 \\
\hline $\begin{array}{c}\text { Cytohchrome C } \\
\text { Peroxidase }\end{array}$ & DTI & 0.299 & 0.296 & 0.281 & 0.275 & 0.273 & 0.229 \\
\hline $\begin{array}{c}\text { Cytohchrome C } \\
\text { Peroxidase }\end{array}$ & ANL & 0.309 & 0.309 & 0.299 & 0.286 & 0.278 & 0.225 \\
\hline $\begin{array}{c}\text { Cytohchrome C } \\
\text { Peroxidase }\end{array}$ & $3 \mathrm{AP}$ & 0.299 & 0.3 & 0.271 & 0.275 & 0.221 & 0.238 \\
\hline $\begin{array}{c}\text { Cytohchrome } \mathrm{C} \\
\text { Peroxidase }\end{array}$ & $4 \mathrm{AP}$ & 0.299 & 0.295 & 0.272 & 0.275 & 0.244 & 0.216 \\
\hline $\begin{array}{c}\text { Cytohchrome } \mathrm{C} \\
\text { Peroxidase }\end{array}$ & $24 \mathrm{~T}$ & 0.299 & 0.298 & 0.271 & 0.275 & 0.229 & 0.227 \\
\hline $\begin{array}{c}\text { Cytohchrome } \mathrm{C} \\
\text { Peroxidase }\end{array}$ & NVI & 0.299 & 0.301 & 0.274 & 0.275 & 0.235 & 0.238 \\
\hline $\begin{array}{c}\text { Cytohchrome } \mathrm{C} \\
\text { Peroxidase }\end{array}$ & IDM & 0.299 & 0.302 & 0.269 & 0.275 & 0.286 & 0.222 \\
\hline $\begin{array}{c}\text { Cytohchrome } \mathrm{C} \\
\text { Peroxidase }\end{array}$ & MPI & 0.299 & 0.301 & 0.276 & 0.275 & 0.289 & 0.195 \\
\hline $\begin{array}{l}\text { Cytohchrome } \mathrm{C} \\
\text { Peroxidase }\end{array}$ & $25 \mathrm{~T}$ & 0.286 & 0.286 & 0.264 & 0.253 & 0.226 & 0.241 \\
\hline $\begin{array}{c}\text { Cytohchrome } \mathrm{C} \\
\text { Peroxidase }\end{array}$ & $2 \mathrm{AP}$ & 0.286 & 0.281 & 0.277 & 0.253 & 0.228 & 0.244 \\
\hline $\begin{array}{l}\text { Cytohchrome } \mathrm{C} \\
\text { Peroxidase }\end{array}$ & $2 \mathrm{EZ}$ & 0.299 & 0.301 & 0.281 & 0.275 & 0.299 & 0.247 \\
\hline $\begin{array}{l}\text { Cytohchrome } \mathrm{C} \\
\text { Peroxidase }\end{array}$ & IMD & 0.31 & 0.318 & 0.241 & 0.285 & 0.285 & 0.221 \\
\hline $\begin{array}{l}\text { Cytohchrome C } \\
\text { Peroxidase }\end{array}$ & $1 \mathrm{MZ}$ & 0.281 & 0.284 & 0.289 & 0.254 & 0.267 & 0.271 \\
\hline $\begin{array}{l}\text { Cytohchrome } \mathrm{C} \\
\text { Peroxidase }\end{array}$ & $2 \mathrm{MZ}$ & 0.286 & 0.293 & 0.228 & 0.253 & 0.269 & 0.156 \\
\hline $\begin{array}{l}\text { Cytohchrome C } \\
\text { Peroxidase } \\
\end{array}$ & AMT & 0.267 & 0.268 & 0.242 & 0.239 & 0.184 & 0.193 \\
\hline $\begin{array}{l}\text { HIV-1 Reverse } \\
\text { Transcriptase }\end{array}$ & FPT & 2.948 & 2.919 & 2.744 & 2.717 & 2.317 & 2.119 \\
\hline $\begin{array}{l}\text { HIV-1 Reverse } \\
\text { Transcriptase }\end{array}$ & FTC & 2.612 & 2.608 & 2.061 & 2.469 & 2.472 & 1.936 \\
\hline $\begin{array}{l}\text { HIV-1 Reverse } \\
\text { Transcriptase }\end{array}$ & EFZ & 2.574 & 2.567 & 2.221 & 2.414 & 2.116 & 2.052 \\
\hline $\begin{array}{l}\text { HIV-1 Reverse } \\
\text { Transcriptase }\end{array}$ & EFZ & 2.554 & 2.553 & 2.03 & 2.405 & 2.419 & 1.853 \\
\hline $\begin{array}{l}\text { HIV-1 Reverse } \\
\text { Transcriptase }\end{array}$ & PNU & 2.419 & 2.426 & 2.287 & 2.212 & 2.227 & 2.103 \\
\hline $\begin{array}{l}\text { HIV-1 Reverse } \\
\text { Transcriptase }\end{array}$ & MSD & 2.413 & 2.398 & 2.656 & 2.214 & 2.394 & 2.221 \\
\hline $\begin{array}{l}\text { HIV-1 Reverse } \\
\text { Transcriptase }\end{array}$ & SBN & 2.452 & 2.443 & 1.947 & 2.344 & 2.114 & 1.856 \\
\hline $\begin{array}{l}\text { HIV-1 Reverse } \\
\text { Transcriptase }\end{array}$ & H12 & 2.982 & 2.975 & 2.652 & 2.791 & 1.884 & 1.783 \\
\hline $\begin{array}{l}\text { HIV-1 Reverse } \\
\text { Transcriptase }\end{array}$ & GWB & 2.384 & 2.403 & 1.978 & 2.24 & 2.198 & 1.572 \\
\hline $\begin{array}{l}\text { HIV-1 Reverse } \\
\text { Transcriptase }\end{array}$ & H16 & 3.002 & 2.986 & 2.725 & 2.806 & 2.774 & 2.659 \\
\hline
\end{tabular}




\begin{tabular}{|c|c|c|c|c|c|c|c|}
\hline $\begin{array}{l}\text { HIV-1 Reverse } \\
\text { Transcriptase }\end{array}$ & $\mathrm{H} 18$ & 2.967 & 2.985 & 2.487 & 2.738 & 2.753 & 2.391 \\
\hline $\begin{array}{l}\text { HIV-1 Reverse } \\
\text { Transcriptase }\end{array}$ & $\mathrm{H} 20$ & 2.947 & 2.959 & 2.682 & 2.731 & 2.447 & 2.512 \\
\hline $\begin{array}{l}\text { HIV-1 Reverse } \\
\text { Transcriptase }\end{array}$ & СР9 & 3.21 & 3.23 & 2.509 & 3.15 & 2.956 & 2.015 \\
\hline $\begin{array}{l}\text { HIV-1 Reverse } \\
\text { Transcriptase }\end{array}$ & $3 \mathrm{AC}$ & 3.183 & 3.225 & 2.411 & 3.113 & 3.118 & 2.289 \\
\hline $\begin{array}{l}\text { HIV-1 Reverse } \\
\text { Transcriptase }\end{array}$ & 357 & 3.516 & 3.535 & 2.317 & 3.41 & 3.128 & 2.115 \\
\hline $\begin{array}{l}\text { HIV-1 Reverse } \\
\text { Transcriptase }\end{array}$ & $\mathrm{R} 22$ & 2.76 & 2.764 & 2.514 & 2.583 & 2.348 & 2.412 \\
\hline $\begin{array}{l}\text { HIV-1 Reverse } \\
\text { Transcriptase }\end{array}$ & NVP & 3.082 & 3.083 & 2.402 & 2.916 & 2.896 & 2.221 \\
\hline $\begin{array}{l}\text { HIV-1 Reverse } \\
\text { Transcriptase }\end{array}$ & RT3 & 2.288 & 2.281 & 2.373 & 2.076 & 2.193 & 1.895 \\
\hline $\begin{array}{l}\text { HIV-1 Reverse } \\
\text { Transcriptase }\end{array}$ & RT7 & 2.074 & 2.089 & 2.494 & 1.813 & 1.956 & 1.882 \\
\hline $\begin{array}{l}\text { HIV-1 Reverse } \\
\text { Transcriptase }\end{array}$ & UDR & 3.692 & 3.681 & 3.058 & 3.594 & 2.297 & 2.114 \\
\hline $\begin{array}{l}\text { HIV-1 Reverse } \\
\text { Transcriptase }\end{array}$ & $\mathrm{AC} 7$ & 3.47 & 3.499 & 2.597 & 3.36 & 2.892 & 1.835 \\
\hline $\begin{array}{l}\text { HIV-1 Reverse } \\
\text { Transcriptase }\end{array}$ & LP9 & 1.918 & 1.92 & 2.233 & 1.66 & 1.472 & 1.491 \\
\hline $\begin{array}{l}\text { HIV-1 Reverse } \\
\text { Transcriptase }\end{array}$ & $65 \mathrm{~B}$ & 2.173 & 2.164 & 2.434 & 1.953 & 1.888 & 1.764 \\
\hline $\begin{array}{l}\text { HIV-1 Reverse } \\
\text { Transcriptase }\end{array}$ & DJZ & 1.992 & 1.982 & 2.806 & 1.752 & 1.645 & 1.889 \\
\hline $\begin{array}{l}\text { HIV-1 Reverse } \\
\text { Transcriptase }\end{array}$ & QO9 & 4.054 & 3.03 & 2.951 & 3.992 & 2.879 & 2.472 \\
\hline Urokinase & ESI & 0.429 & 0.423 & 0.325 & 0.376 & 0.387 & 0.286 \\
\hline Urokinase & ESP & 0.43 & 0.43 & 0.341 & 0.379 & 0.336 & 0.297 \\
\hline Urokinase & BAM & 0.44 & 0.449 & 0.299 & 0.389 & 0.338 & 0.241 \\
\hline Urokinase & AGB & 0.404 & 0.408 & 0.376 & 0.376 & 0.346 & 0.328 \\
\hline Urokinase & BAM & 0.377 & 0.381 & 0.312 & 0.338 & 0.375 & 0.266 \\
\hline Urokinase & AMR & 0.379 & 0.379 & 0.392 & 0.345 & 0.318 & 0.327 \\
\hline Urokinase & UKP & 0.397 & 0.401 & 0.364 & 0.349 & 0.389 & 0.314 \\
\hline Urokinase & 120 & 0.446 & 0.451 & 0.381 & 0.391 & 0.442 & 0.294 \\
\hline Urokinase & BMZ & 0.429 & 0.428 & 0.379 & 0.37 & 0.326 & 0.318 \\
\hline Urokinase & 123 & 0.43 & 0.427 & 0.354 & 0.383 & 0.368 & 0.296 \\
\hline Urokinase & 132 & 0.409 & 0.41 & 0.328 & 0.367 & 0.389 & 0.296 \\
\hline Urokinase & 133 & 0.416 & 0.408 & 0.346 & 0.363 & 0.383 & 0.317 \\
\hline
\end{tabular}




\begin{tabular}{|l|l|l|l|l|l|l|l|} 
Urokinase & 135 & 0.396 & 0.396 & 0.319 & 0.348 & 0.305 & 0.264 \\
\hline Urokinase & 130 & 0.465 & 0.457 & 0.379 & 0.417 & 0.413 & 0.329 \\
\hline Urokinase & 130 & 0.431 & 0.444 & 0.365 & 0.389 & 0.385 & 0.344 \\
\hline Urokinase & 136 & 0.405 & 0.402 & 0.371 & 0.352 & 0.393 & 0.308 \\
\hline Urokinase & 655 & 0.438 & 0.431 & 0.349 & 0.39 & 0.372 & 0.314 \\
\hline Urokinase & 696 & 0.416 & 0.408 & 0.361 & 0.37 & 0.389 & 0.294 \\
\hline Urokinase & ESI & 0.42 & 0.413 & 0.374 & 0.375 & 0.367 & 0.312 \\
\hline Urokinase & CR9 & 0.408 & 0.403 & 0.341 & 0.357 & 0.388 & 0.295 \\
\hline
\end{tabular}

Table S6. Side-chain and all-atom RMSD $(\AA)$ for the binding pocket residues between the bound and the docked pose obtained from 'Flexible-backbone' protocol docking of unbound protein with RosettaLigand, 'Adaptive BP-Dock' docking of unbound protein along the with the side-chain rmsd of those between unbound and bound experimental structure as a reference.

\begin{tabular}{|c|c|c|c|c|c|c|c|}
\hline \multirow[b]{2}{*}{ Protein } & \multirow[b]{2}{*}{$\begin{array}{l}\text { Peptide/ } \\
\text { Ligand }\end{array}$} & \multicolumn{3}{|c|}{$\mathbf{R M S D}_{\text {sc-BindingPocket }}(\AA)$} & \multicolumn{3}{|c|}{$\mathbf{R M S D}_{\text {allatom-BindingPocket }}(\mathbf{\AA})$} \\
\hline & & $\begin{array}{l}\text { Bound- } \\
\text { Unbound }\end{array}$ & $\begin{array}{l}\text { Bound- } \\
\text { Rosetta } \\
\text { Ligand } \\
\text { Unbound } \\
\text { Docked } \\
\text { Pose }\end{array}$ & $\begin{array}{c}\text { Bound- } \\
\text { Adaptive } \\
\text { BP-Dock } \\
\text { Unbound } \\
\text { Docked } \\
\text { Pose }\end{array}$ & $\begin{array}{l}\text { Bound- } \\
\text { Unbound }\end{array}$ & $\begin{array}{l}\text { Bound- } \\
\text { Rosetta } \\
\text { Ligand } \\
\text { Unbound } \\
\text { Docked } \\
\text { Pose }\end{array}$ & $\begin{array}{c}\text { Bound- } \\
\text { Adaptive } \\
\text { BP-Dock } \\
\text { Unbound } \\
\text { Docked } \\
\text { Pose }\end{array}$ \\
\hline HIV-Protease & SB203238 & 0.872 & 0.892 & 0.784 & 0.818 & 0.764 & 0.723 \\
\hline HIV-Protease & SKF 107457 & 1.01 & 1.162 & 0.927 & 0.894 & 0.819 & 0.794 \\
\hline HIV-Protease & CGP-53820 & 0.841 & 0.672 & 0.643 & 0.695 & 0.662 & 0.627 \\
\hline HIV-Protease & U75875 & 0.822 & 1.006 & 0.798 & 0.758 & 0.735 & 0.719 \\
\hline HIV-Protease & SB206343 & 1.155 & 1.038 & 1.024 & 0.91 & 0.892 & 0.885 \\
\hline HIV-Protease & GR123976 & 0.966 & 0.976 & 0.893 & 0.634 & 0.547 & 0.498 \\
\hline HIV-Protease & GR126045 & 0.955 & 0.927 & 0.934 & 0.871 & 0.889 & 0.803 \\
\hline HIV-Protease & GR137615 & 0.82 & 0.844 & 0.781 & 0.631 & 0.601 & 0.574 \\
\hline HIV-Protease & A77003 (R,S) & 0.78 & 0.8 & 0.656 & 0.712 & 0.781 & 0.561 \\
\hline HIV-Protease & A78791 (S,-) & 0.793 & 1 & 0.782 & 0.678 & 0.62 & 0.618 \\
\hline HIV-Protease & A76928 (S,S) & 0.785 & 1.09 & 0.773 & 0.746 & 0.738 & 0.681 \\
\hline HIV-Protease & A76889 (R,R) & 0.824 & 0.807 & 0.683 & 0.698 & 0.705 & 0.629 \\
\hline HIV-Protease & A77 & 0.795 & 1.195 & 0.701 & 0.712 & 0.981 & 0.68 \\
\hline HIV-Protease & IM1 & 0.954 & 0.888 & 0.865 & 0.899 & 0.811 & 0.735 \\
\hline HIV-Protease & MVT-101 & 0.688 & 0.735 & 0.502 & 0.711 & 0.691 & 0.627 \\
\hline HIV-Protease & L-700, 417 & 0.821 & 0.81 & 0.798 & 0.663 & 0.794 & 0.467 \\
\hline
\end{tabular}




\begin{tabular}{|c|c|c|c|c|c|c|c|}
\hline HIV-Protease & $\begin{array}{c}\text { ACETYL- } \\
\text { PEPSTATIN }\end{array}$ & 1.247 & 1.123 & 0.983 & 0.75 & 0.813 & 0.781 \\
\hline HIV-Protease & A-74704 & 1.272 & 1.352 & 1.112 & 1.082 & 1.191 & 0.992 \\
\hline HIV-Protease & Tri-peptide & 0.786 & 0.748 & 0.729 & 0.774 & 0.629 & 0.571 \\
\hline HIV-Protease & JE-2147 & 0.832 & 0.911 & 0.771 & 0.712 & 0.816 & 0.619 \\
\hline $\begin{array}{c}\text { Carbonic } \\
\text { Anhydrase II }\end{array}$ & Celecoxib & 0.248 & 0.272 & 0.221 & 0.239 & 0.223 & 0.208 \\
\hline $\begin{array}{c}\text { Carbonic } \\
\text { Anhydrase II }\end{array}$ & Histamine & 0.144 & 0.286 & 0.169 & 0.14 & 0.257 & 0.139 \\
\hline $\begin{array}{c}\text { Carbonic } \\
\text { Anhydrase II }\end{array}$ & ETS & 0.255 & 0.263 & 0.245 & 0.236 & 0.212 & 0.225 \\
\hline $\begin{array}{c}\text { Carbonic } \\
\text { Anhydrase II }\end{array}$ & PTS & 0.188 & 0.398 & 0.192 & 0.174 & 0.181 & 0.145 \\
\hline $\begin{array}{c}\text { Carbonic } \\
\text { Anhydrase II } \\
\end{array}$ & MTS & 0.144 & 0.369 & 0.148 & 0.132 & 0.347 & 0.116 \\
\hline $\begin{array}{c}\text { Carbonic } \\
\text { Anhydrase II }\end{array}$ & EG1 & 0.124 & 0.318 & 0.119 & 0.118 & 0.235 & 0.091 \\
\hline $\begin{array}{c}\text { Carbonic } \\
\text { Anhydrase II } \\
\end{array}$ & EG2 & 0.183 & 0.29 & 0.173 & 0.109 & 0.198 & 0.102 \\
\hline $\begin{array}{c}\text { Carbonic } \\
\text { Anhydrase II }\end{array}$ & EG3 & 0.233 & 0.297 & 0.194 & 0.226 & 0.214 & 0.173 \\
\hline $\begin{array}{c}\text { Carbonic } \\
\text { Anhydrase II }\end{array}$ & MNS & 0.241 & 0.223 & 0.204 & 0.237 & 0.194 & 0.115 \\
\hline $\begin{array}{c}\text { Alcohol } \\
\text { Deydrogenase }\end{array}$ & CND & 1.156 & 1.015 & 1.125 & 0.98 & 0.963 & 0.924 \\
\hline $\begin{array}{c}\text { Alcohol } \\
\text { Deydrogenase }\end{array}$ & PAD & 1.218 & 1.092 & 1.221 & 0.95 & 0.814 & 0.993 \\
\hline $\begin{array}{c}\text { Alcohol } \\
\text { Deydrogenase }\end{array}$ & TAD & 0.446 & 0.495 & 0.427 & 0.415 & 0.291 & 0.341 \\
\hline $\begin{array}{c}\text { Alcohol } \\
\text { Deydrogenase }\end{array}$ & SSB & 1.004 & 1.079 & 0.986 & 0.973 & 0.827 & 0.715 \\
\hline $\begin{array}{c}\text { Alcohol } \\
\text { Deydrogenase }\end{array}$ & $\mathrm{PFB}, \mathrm{NAD}$ & 1.047 & 1.232 & 1.012 & 0.97 & 0.991 & 0.836 \\
\hline $\begin{array}{c}\text { Alcohol } \\
\text { Deydrogenase }\end{array}$ & FPI,NAD & 1.031 & 1.201 & 0.986 & 0.937 & 0.916 & 0.845 \\
\hline $\begin{array}{c}\text { Alcohol } \\
\text { Deydrogenase }\end{array}$ & CXF,NAD & 1.038 & 1.017 & 0.973 & 0.978 & 0.896 & 0.771 \\
\hline $\begin{array}{c}\text { Alcohol } \\
\text { Deydrogenase }\end{array}$ & $\mathrm{SSB}, \mathrm{NAD}$ & 1.067 & 1.083 & 0.992 & 0.964 & 0.874 & 0.849 \\
\hline $\begin{array}{c}\text { Alpha- } \\
\text { Thrombin }\end{array}$ & QWE & 0.327 & 0.378 & 0.226 & 0.318 & 0.228 & 0.185 \\
\hline $\begin{array}{c}\text { Alpha- } \\
\text { Thrombin }\end{array}$ & AZL & 0.231 & 0.362 & 0.249 & 0.225 & 0.314 & 0.201 \\
\hline $\begin{array}{c}\text { Alpha- } \\
\text { Thrombin }\end{array}$ & BMS-186282 & 0.609 & 0.601 & 0.591 & 0.502 & 0.569 & 0.419 \\
\hline $\begin{array}{c}\text { Alpha- } \\
\text { Thrombin }\end{array}$ & BMS-189090 & 0.415 & 0.379 & 0.422 & 0.393 & 0.336 & 0.293 \\
\hline $\begin{array}{c}\text { Alpha- } \\
\text { Thrombin }\end{array}$ & BZT & 0.668 & 0.662 & 0.651 & 0.554 & 0.561 & 0.484 \\
\hline $\begin{array}{c}\text { Alpha- } \\
\text { Thrombin }\end{array}$ & BT3 & 0.667 & 0.634 & 0.619 & 0.593 & 0.582 & 0.468 \\
\hline $\begin{array}{c}\text { Alpha- } \\
\text { Thrombin }\end{array}$ & BPP & 0.32 & 0.395 & 0.235 & 0.296 & 0.229 & 0.193 \\
\hline $\begin{array}{c}\text { Alpha- } \\
\text { Thrombin }\end{array}$ & $\begin{array}{c}\text { Benzyldiamin } \\
\mathrm{e} \\
\end{array}$ & 0.229 & 0.337 & 0.212 & 0.201 & 0.284 & 0.146 \\
\hline $\begin{array}{c}\text { Alpha- } \\
\text { Thrombin }\end{array}$ & $\begin{array}{c}\text { MD-805 } \\
\text { (Argatroban) }\end{array}$ & 0.297 & 0.326 & 0.218 & 0.294 & 0.249 & 0.154 \\
\hline $\begin{array}{c}\text { Alpha- } \\
\text { Thrombin }\end{array}$ & NAPAP & 0.176 & 0.325 & 0.104 & 0.163 & 0.256 & 0.056 \\
\hline $\begin{array}{c}\text { Alpha- } \\
\text { Thrombin }\end{array}$ & BMS-183507 & 0.485 & 0.701 & 0.385 & 0.425 & 0.613 & 0.249 \\
\hline $\begin{array}{c}\text { Alpha- } \\
\text { Thrombin }\end{array}$ & BM51.1011 & 0.439 & 0.426 & 0.371 & 0.418 & 0.381 & 0.267 \\
\hline
\end{tabular}




\begin{tabular}{|c|c|c|c|c|c|c|c|}
\hline $\begin{array}{c}\text { Alpha- } \\
\text { Thrombin }\end{array}$ & FSN & 0.386 & 0.376 & 0.229 & 0.338 & 0.319 & 0.173 \\
\hline $\begin{array}{c}\text { Cytohchrome } \mathrm{C} \\
\text { Peroxidase }\end{array}$ & TMT & 0.377 & 0.411 & 0.389 & 0.361 & 0.371 & 0.329 \\
\hline $\begin{array}{c}\text { Cytohchrome } \mathrm{C} \\
\text { Peroxidase } \\
\end{array}$ & TMZ & 0.465 & 0.502 & 0.423 & 0.448 & 0.442 & 0.381 \\
\hline $\begin{array}{c}\text { Cytohchrome C } \\
\text { Peroxidase }\end{array}$ & $3 \mathrm{MT}$ & 0.461 & 0.494 & 0.452 & 0.451 & 0.415 & 0.393 \\
\hline $\begin{array}{c}\text { Cytohchrome } \mathrm{C} \\
\text { Peroxidase } \\
\end{array}$ & DTI & 0.461 & 0.516 & 0.438 & 0.452 & 0.447 & 0.376 \\
\hline $\begin{array}{c}\text { Cytohchrome } \mathrm{C} \\
\text { Peroxidase }\end{array}$ & ANL & 0.471 & 0.499 & 0.442 & 0.46 & 0.387 & 0.331 \\
\hline $\begin{array}{c}\text { Cytohchrome } \mathrm{C} \\
\text { Peroxidase } \\
\end{array}$ & $3 \mathrm{AP}$ & 0.461 & 0.479 & 0.439 & 0.452 & 0.379 & 0.317 \\
\hline $\begin{array}{c}\text { Cytohchrome } \mathrm{C} \\
\text { Peroxidase } \\
\end{array}$ & $4 \mathrm{AP}$ & 0.461 & 0.49 & 0.445 & 0.451 & 0.368 & 0.291 \\
\hline $\begin{array}{c}\text { Cytohchrome } \mathrm{C} \\
\text { Peroxidase } \\
\end{array}$ & $24 \mathrm{~T}$ & 0.462 & 0.503 & 0.431 & 0.451 & 0.422 & 0.454 \\
\hline $\begin{array}{c}\text { Cytohchrome } \mathrm{C} \\
\text { Peroxidase } \\
\end{array}$ & NVI & 0.461 & 0.495 & 0.428 & 0.45 & 0.448 & 0.378 \\
\hline $\begin{array}{c}\text { Cytohchrome C } \\
\text { Peroxidase }\end{array}$ & IDM & 0.463 & 0.494 & 0.391 & 0.451 & 0.417 & 0.342 \\
\hline $\begin{array}{c}\text { Cytohchrome } \mathrm{C} \\
\text { Peroxidase }\end{array}$ & MPI & 0.461 & 0.503 & 0.413 & 0.452 & 0.451 & 0.357 \\
\hline $\begin{array}{c}\text { Cytohchrome C } \\
\text { Peroxidase }\end{array}$ & $25 \mathrm{~T}$ & 0.377 & 0.419 & 0.318 & 0.359 & 0.339 & 0.271 \\
\hline $\begin{array}{c}\text { Cytohchrome } \mathrm{C} \\
\text { Peroxidase }\end{array}$ & $2 \mathrm{AP}$ & 0.377 & 0.427 & 0.297 & 0.362 & 0.252 & 0.118 \\
\hline $\begin{array}{l}\text { Cytohchrome C } \\
\text { Peroxidase }\end{array}$ & $2 \mathrm{EZ}$ & 0.464 & 0.497 & 0.469 & 0.423 & 0.377 & 0.317 \\
\hline $\begin{array}{c}\text { Cytohchrome C } \\
\text { Peroxidase }\end{array}$ & IMD & 0.416 & 0.458 & 0.375 & 0.398 & 0.256 & 0.319 \\
\hline $\begin{array}{l}\text { Cytohchrome } \mathrm{C} \\
\text { Peroxidase }\end{array}$ & $1 \mathrm{MZ}$ & 0.42 & 0.471 & 0.391 & 0.418 & 0.439 & 0.295 \\
\hline $\begin{array}{c}\text { Cytohchrome C } \\
\text { Peroxidase }\end{array}$ & $2 \mathrm{MZ}$ & 0.377 & 0.422 & 0.348 & 0.335 & 0.308 & 0.226 \\
\hline $\begin{array}{c}\text { Cytohchrome C } \\
\text { Peroxidase }\end{array}$ & AMT & 0.431 & 0.472 & 0.402 & 0.401 & 0.271 & 0.318 \\
\hline $\begin{array}{l}\text { HIV-1 Reverse } \\
\text { Transcriptase }\end{array}$ & FPT & 3.022 & 2.76 & 2.246 & 2.985 & 2.117 & 1.783 \\
\hline $\begin{array}{c}\text { HIV-1 Reverse } \\
\text { Transcriptase }\end{array}$ & FTC & 5.807 & 5.675 & 5.271 & 5.581 & 4.579 & 4.291 \\
\hline $\begin{array}{l}\text { HIV-1 Reverse } \\
\text { Transcriptase }\end{array}$ & EFZ & 3.782 & 3.72 & 3.178 & 3.101 & 3.296 & 2.694 \\
\hline $\begin{array}{l}\text { HIV-1 Reverse } \\
\text { Transcriptase }\end{array}$ & EFZ & 2.442 & 2.495 & 2.042 & 1.848 & 1.962 & 1.663 \\
\hline $\begin{array}{l}\text { HIV-1 Reverse } \\
\text { Transcriptase }\end{array}$ & PNU & 2.453 & 2.376 & 1.984 & 1.696 & 2.003 & 1.025 \\
\hline $\begin{array}{l}\text { HIV-1 Reverse } \\
\text { Transcriptase }\end{array}$ & MSD & 2.242 & 2.308 & 2.219 & 1.549 & 2.199 & 1.782 \\
\hline $\begin{array}{c}\text { HIV-1 Reverse } \\
\text { Transcriptase }\end{array}$ & SBN & 0.553 & 0.553 & 0.519 & 1.664 & 0.471 & 0.337 \\
\hline $\begin{array}{l}\text { HIV-1 Reverse } \\
\text { Transcriptase }\end{array}$ & H12 & 0.269 & 0.718 & 0.264 & 1.033 & 0.568 & 0.137 \\
\hline $\begin{array}{l}\text { HIV-1 Reverse } \\
\text { Transcriptase }\end{array}$ & GWB & 2.621 & 2.069 & 2.619 & 1.856 & 1.278 & 1.887 \\
\hline $\begin{array}{l}\text { HIV-1 Reverse } \\
\text { Transcriptase }\end{array}$ & H16 & 0.026 & 0.041 & 0.028 & 0.257 & 0.013 & 0.011 \\
\hline $\begin{array}{l}\text { HIV-1 Reverse } \\
\text { Transcriptase }\end{array}$ & H18 & 0.03 & 0.02 & 0.017 & 0.218 & 0.022 & 0.019 \\
\hline $\begin{array}{c}\text { HIV-1 Reverse } \\
\text { Transcriptase }\end{array}$ & $\mathrm{H} 20$ & 0.019 & 0.021 & 0.014 & 0.232 & 0.118 & 0.114 \\
\hline $\begin{array}{l}\text { HIV-1 Reverse } \\
\text { Transcriptase }\end{array}$ & СР9 & 0.928 & 1.157 & 0.457 & 0.557 & 0.791 & 0.327 \\
\hline
\end{tabular}




\begin{tabular}{|c|c|c|c|c|c|c|c|}
\hline $\begin{array}{l}\text { HIV-1 Reverse } \\
\text { Transcriptase }\end{array}$ & $3 \mathrm{AC}$ & 1.215 & 1.318 & 0.986 & 1.04 & 0.937 & 0.661 \\
\hline $\begin{array}{l}\text { HIV-1 Reverse } \\
\text { Transcriptase }\end{array}$ & 357 & 2.418 & 3.043 & 1.774 & 1.67 & 1.01 & 0.994 \\
\hline $\begin{array}{l}\text { HIV-1 Reverse } \\
\text { Transcriptase }\end{array}$ & R22 & 1.025 & 1.311 & 0.893 & 0.818 & 0.979 & 0.753 \\
\hline $\begin{array}{l}\text { HIV-1 Reverse } \\
\text { Transcriptase }\end{array}$ & NVP & 3.22 & 3.098 & 2.348 & 3.484 & 2.381 & 1.941 \\
\hline $\begin{array}{l}\text { HIV-1 Reverse } \\
\text { Transcriptase }\end{array}$ & RT3 & 5.067 & 5.117 & 4.812 & 4.993 & 3.489 & 2.261 \\
\hline $\begin{array}{l}\text { HIV-1 Reverse } \\
\text { Transcriptase }\end{array}$ & RT7 & 5.119 & 5.347 & 4.17 & 4.872 & 4.168 & 3.631 \\
\hline $\begin{array}{l}\text { HIV-1 Reverse } \\
\text { Transcriptase }\end{array}$ & UDR & 4.96 & 5.154 & 5.024 & 4.599 & 4.11 & 3.988 \\
\hline $\begin{array}{l}\text { HIV-1 Reverse } \\
\text { Transcriptase }\end{array}$ & $\mathrm{AC7}$ & 2.079 & 1.652 & 1.205 & 1.369 & 1.391 & 1.013 \\
\hline $\begin{array}{l}\text { HIV-1 Reverse } \\
\text { Transcriptase }\end{array}$ & LP9 & 6.723 & 6.281 & 6.392 & 5.392 & 4.268 & 4.481 \\
\hline $\begin{array}{l}\text { HIV-1 Reverse } \\
\text { Transcriptase }\end{array}$ & $65 \mathrm{~B}$ & 6.582 & 6.692 & 5.279 & 5.482 & 3.411 & 3.985 \\
\hline $\begin{array}{l}\text { HIV-1 Reverse } \\
\text { Transcriptase }\end{array}$ & DJZ & 3.61 & 3.495 & 3.792 & 3.732 & 2.469 & 2.228 \\
\hline $\begin{array}{l}\text { HIV-1 Reverse } \\
\text { Transcriptase }\end{array}$ & QO9 & 5.678 & 5.741 & 4.916 & 5.456 & 4.279 & 3.935 \\
\hline Urokinase & ESI & 0.278 & 0.265 & 0.212 & 0.275 & 0.189 & 0.165 \\
\hline Urokinase & ESP & 0.266 & 0.288 & 0.248 & 0.291 & 0.227 & 0.201 \\
\hline Urokinase & BAM & 0.319 & 0.271 & 0.282 & 0.305 & 0.229 & 0.248 \\
\hline Urokinase & AGB & 0.239 & 0.22 & 0.198 & 0.249 & 0.185 & 0.163 \\
\hline Urokinase & BAM & 0.182 & 0.255 & 0.164 & 0.21 & 0.22 & 0.126 \\
\hline Urokinase & AMR & 0.242 & 0.311 & 0.192 & 0.245 & 0.295 & 0.173 \\
\hline Urokinase & UKP & 0.263 & 0.205 & 0.185 & 0.258 & 0.117 & 0.148 \\
\hline Urokinase & 120 & 0.348 & 0.315 & 0.256 & 0.289 & 0.274 & 0.187 \\
\hline Urokinase & BMZ & 0.349 & 0.329 & 0.312 & 0.273 & 0.283 & 0.226 \\
\hline Urokinase & 123 & 0.441 & 0.394 & 0.34 & 0.313 & 0.27 & 0.291 \\
\hline Urokinase & 132 & 0.338 & 0.306 & 0.302 & 0.313 & 0.287 & 0.225 \\
\hline Urokinase & 133 & 0.356 & 0.27 & 0.288 & 0.326 & 0.193 & 0.185 \\
\hline Urokinase & 135 & 0.415 & 0.287 & 0.351 & 0.282 & 0.151 & 0.223 \\
\hline Urokinase & 130 & 0.339 & 0.316 & 0.293 & 0.294 & 0.263 & 0.222 \\
\hline Urokinase & 130 & 0.388 & 0.256 & 0.194 & 0.304 & 0.168 & 0.115 \\
\hline Urokinase & 136 & 0.26 & 0.225 & 0.116 & 0.267 & 0.185 & 0.093 \\
\hline Urokinase & 655 & 0.401 & 0.321 & 0.385 & 0.393 & 0.245 & 0.227 \\
\hline Urokinase & 696 & 0.359 & 0.258 & 0.222 & 0.338 & 0.206 & 0.168 \\
\hline Urokinase & ESI & 0.301 & 0.251 & 0.239 & 0.299 & 0.192 & 0.164 \\
\hline Urokinase & CR9 & 0.329 & 0.241 & 0.237 & 0.317 & 0.192 & 0.153 \\
\hline
\end{tabular}




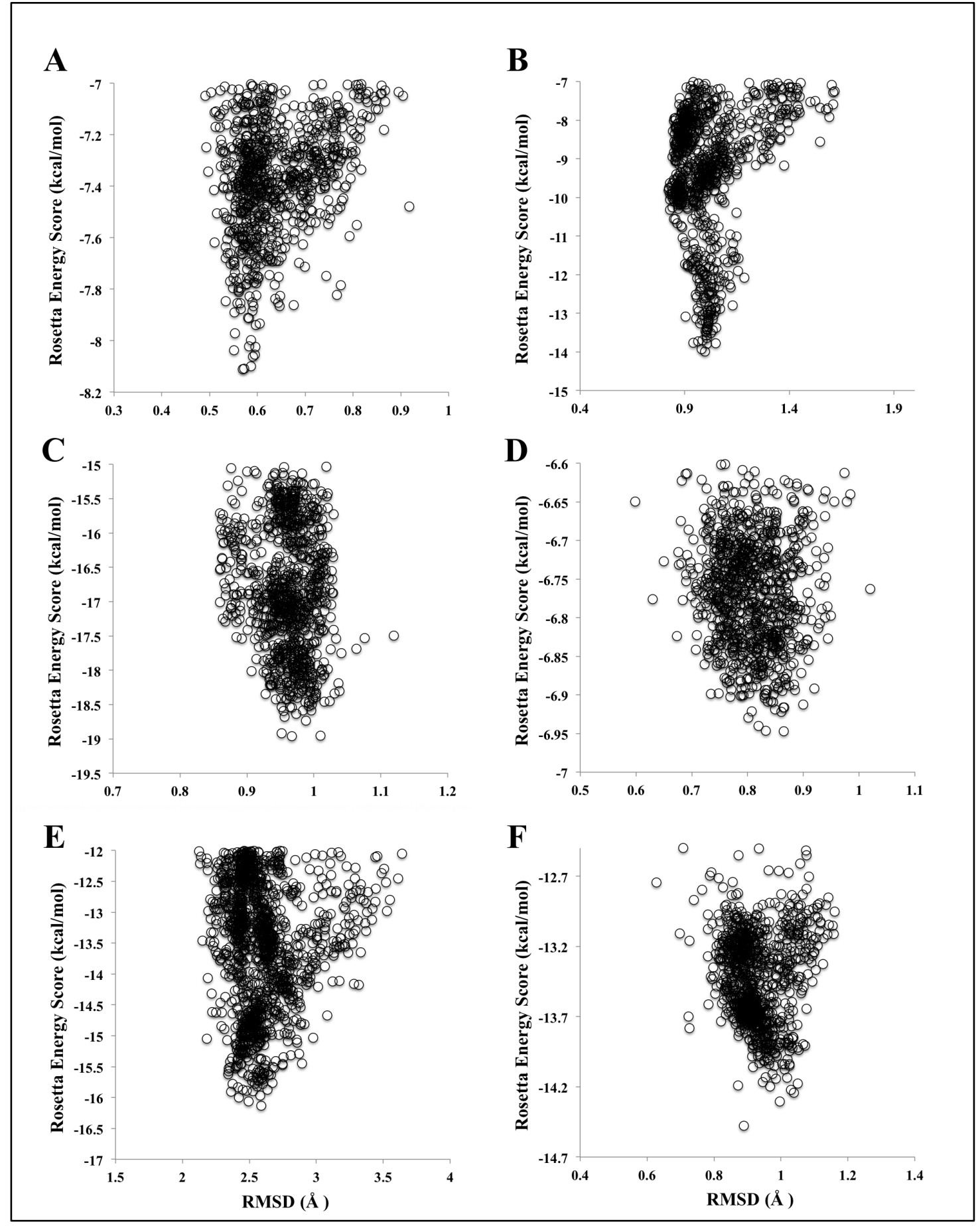

Figure S1. Energy score $(\mathrm{kcal} / \mathrm{mol})$ vs. RMSD $\mathrm{D}_{\text {ligand }}(\AA)$ plots for Adaptive BP-Dock docking of unbound (A) Carbonic Anhydrase II (ligand from 1CIN), (B) Alcohol Dehydrogenase (ligand from 1ADB), (C) Alpha-Thrombin (ligand from 1HDT), (D) Cytochrome $\mathrm{C}$ Peroxidase (ligand from 1AEH), (E) HIV-1 Reverse Transcriptase (ligand from 2BAN) and (F) Urokinase (ligand from 1GJA) proteins. 


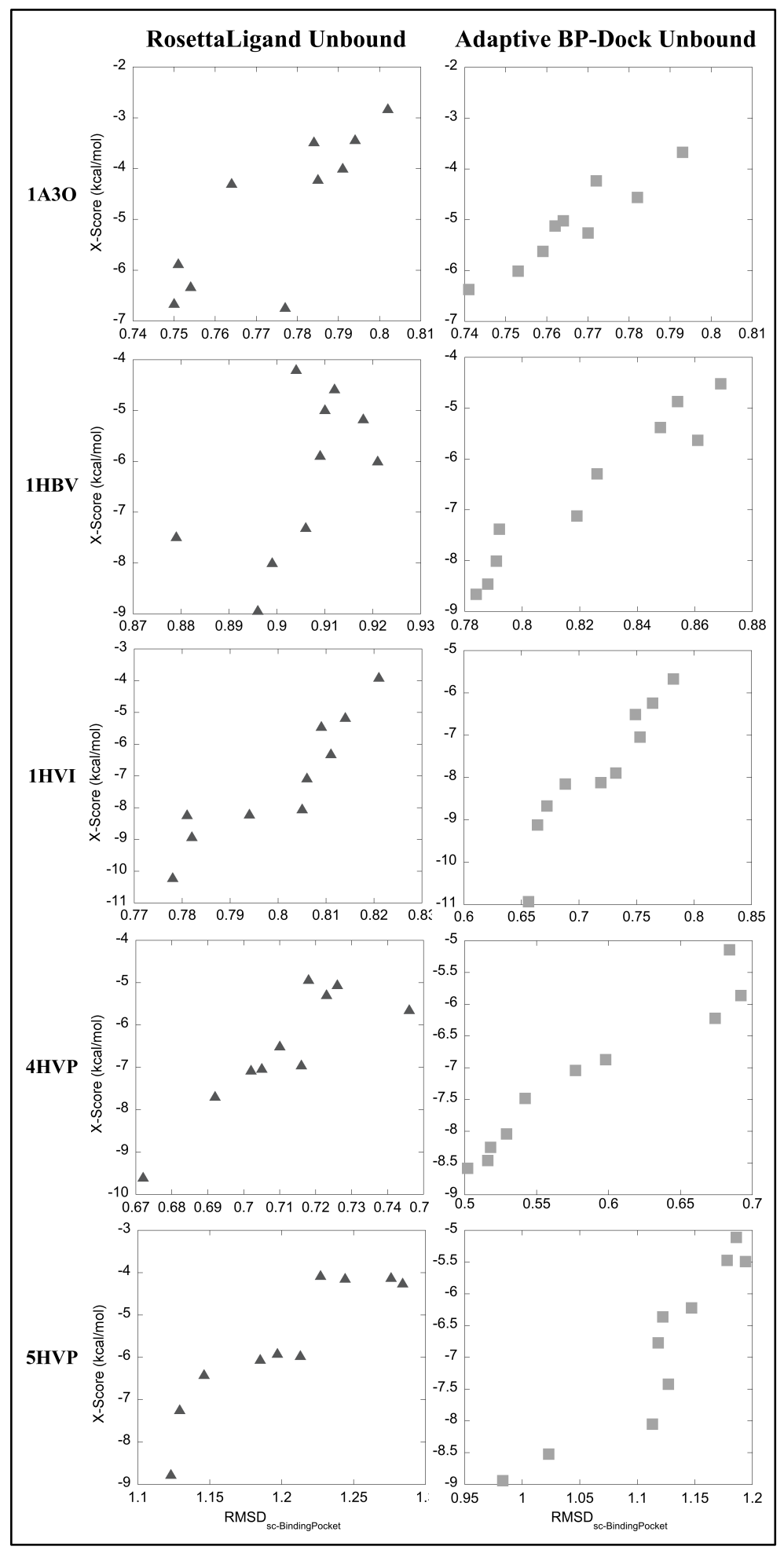

Figure S2. Side-chain RMSD $(\AA)$ for the binding pocket residues vs. binding energy scores (X-Score) for each iteration 1-10 for RosettaLigand Unbound and Adaptive BP-Dock unbound docking for five HIV-1 Protease proteins. 


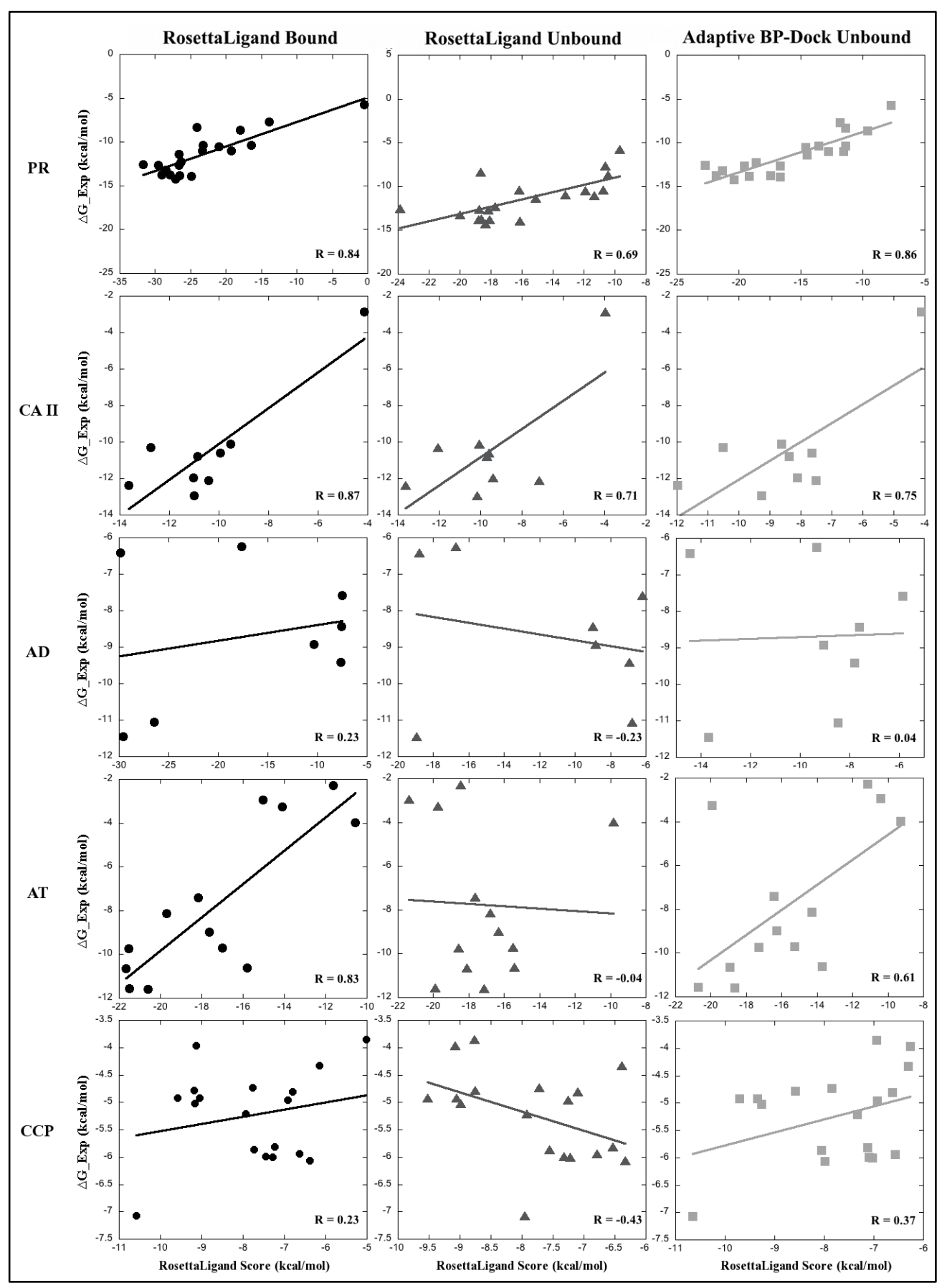

Figure S3. Correlation plots of RosettaLigand Interface Delta energy scores vs. experimental binding energies for HIV-1 Protease (PR), Carbonic Anhydrase II (CA II), Alcohol Dehydrogenase (AD), Alpha-Thrombin (AT) and Cytochrome C Peroxidase (CCP). 


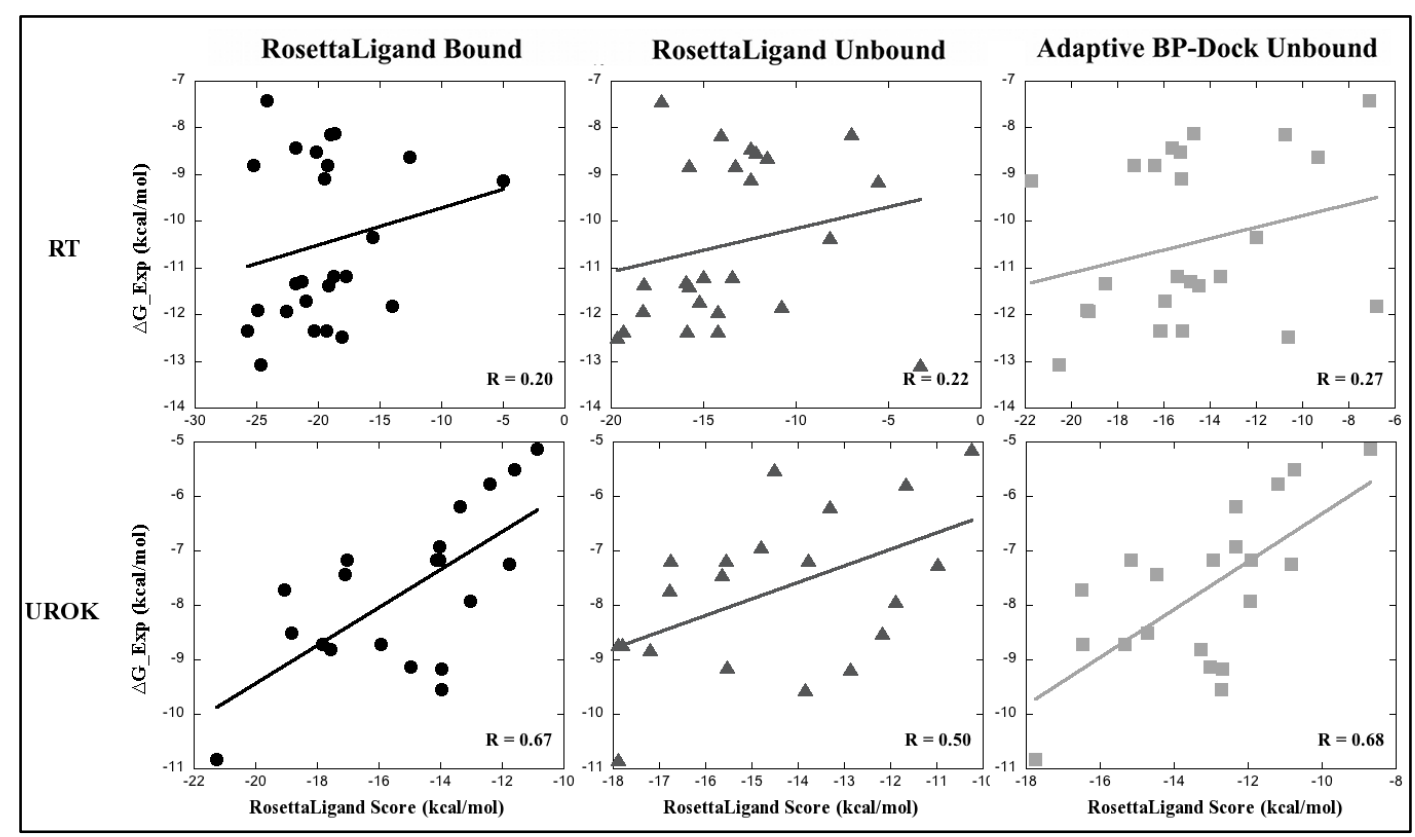

Figure S4. Correlation plots of RosettaLigand Interface Delta energy scores $v s$. experimental binding energies for HIV-1 Reverse Transcriptase (RT), and Urokinase (UROK). 